\title{
Multi-loop Pilot Behaviour Identification in Response to Simultaneous Visual and Haptic Stimuli
}

\author{
Mario Olivari* \\ Max Planck Institute for Biological Cybernetics, Tübingen, Germany \\ University of Pisa, 56126 Pisa, Italy \\ Frank M. Nieuwenhuizen ${ }^{\dagger}$ and Joost Venrooij ${ }^{\ddagger}$ \\ Max Planck Institute for Biological Cybernetics, Tübingen, Germany \\ Delft University of Technology, Delft, The Netherlands \\ Heinrich H. Bülthoff ${ }^{\S}$ \\ Max Planck Institute for Biological Cybernetics, Tübingen, Germany \\ Department of Brain and Cognitive Engineering, Korea University, Seoul, South Korea \\ and \\ Lorenzo Pollini \\ Department of Electrical Systems and Automation, University of Pisa, Pisa, Italy
}

\begin{abstract}
The goal of this paper is to better understand how the neuromuscular system of a pilot, or more generally an operator, adapts itself to different types of haptic aids during a pitch control task. A multi-loop pilot model, capable of describing the human behaviour during a tracking task, is presented. Three different identification techniques were investigated in order to simultaneously identify neuromuscular admittance and the visual response of a human pilot. In one of them, the various frequency response functions that build up the pilot model are identified using multi-inputs linear time-invariant models in ARX form. A second method makes use of cross-spectral densities and diagram block algebra to obtain the desired frequency response estimates. The identification techniques were validated using Monte Carlo simulations of a closed-loop control task. Both techniques were compared with the results of another identification method well known in literature and based on crossspectral density estimates. All those methods were applied in an experimental setup in which pilots performed a pitch control task with different haptic aids. Two different haptic aids for tracking task are presented, a Direct Haptic Aid and an Indirect Haptic Aid. The two haptic aids were compared with a baseline condition in which no haptic force was used. The data obtained with the proposed method provide insight in how the pilot adapts his control behavior in relation to different haptic feedback schemes. From the experimental results it can be concluded that humans adapt their neuromuscular admittance in relation with different haptic aids. Furthermore, the two new identification techniques seemed to give more reliable admittance estimates.
\end{abstract}

\footnotetext{
* Master thesis student, Max Planck Institute for Biological Cybernetics, P.O. Box 2169, 72076 Tübingen, Germany; mario.olivari@tuebingen.mpg.de.

${ }^{\dagger}$ Research Scientist, Max Planck Institute for Biological Cybernetics, P.O Box 2169, 72076 Tübingen, Germany; frank.nieuwenhuizen@tuebingen.mpg.de. Student Member AIAA.

$\ddagger$ Ph.D. student, Max Planck Institute for Biological Cybernetics, P.O Box 2169, 72076 Tübingen, Germany; joost.venrooij@tuebingen.mpg.de.

$\S$ Professor and Director, Max Planck Institute for Biological Cybernetics, P.O Box 2169, 72076 Tübingen, Germany; heinrich.buelthoff@tuebingen.mpg.de. Adjunct Professor, Department of Brain and Cognitive Engineering, Korea University, Seoul, South Korea. Member AIAA.

IAssistant Professor, Department Electrical Systems and Automations, Faculty of Automation Engineering, Pisa, Italy; Lorenzo.Pollini@dsea.unipi.it. Senior Member AIAA.
} 


\section{Nomenclature}

\begin{tabular}{|c|c|c|c|}
\hline א. & Coherence function. & $H_{f}$ & Filter for $x_{t a r}$ amplitude. \\
\hline$\hat{S}_{w w}$ & Square spectral density estimate of & $H_{n}$ & Remnant filter dynamics. \\
\hline & generic signal $w$. & $H_{p}$ & Pilot lumped dynamics. \\
\hline$\hat{S}_{w v}$ & $\begin{array}{l}\text { Cross-spectral density estimate of } \\
\text { generic signals } w \text { and } v \text {. }\end{array}$ & $\begin{array}{l}H_{a d m} \\
H_{C D}\end{array}$ & $\begin{array}{l}\text { Admittance dynamics. } \\
\text { Control device transfer function. }\end{array}$ \\
\hline$\omega_{c}$ & Crossover frequency $(\mathrm{Hz})$. & $H_{C E}$ & Controlled element transfer function. \\
\hline e & Equivalent time delay (s). & $H_{\text {dist }}$ & Transfer function from $F_{\text {dist }}$ to $x_{C D}$ \\
\hline & Visual error (rad). & $H_{O L}$ & Open-loop dynamics. \\
\hline$f_{d}$ & $\begin{array}{l}\text { Force disturbance set of frequencies } \\
(\mathrm{Hz}) \text {. }\end{array}$ & $\begin{array}{l}H_{\text {tar }} \\
H_{\text {vis }}\end{array}$ & $\begin{array}{l}\text { Transfer function from } x_{t a r} \text { to } x_{C D} \\
\text { Visual response dynamics. }\end{array}$ \\
\hline$f_{t}$ & Target position set of frequencies $(\mathrm{Hz})$. & $N$ & Pilot remnant $(\mathrm{N})$ \\
\hline$F_{a d m}$ & $\begin{array}{l}\text { Human force given by arm admittance } \\
(\mathrm{N}) \text {. }\end{array}$ & $u$ & $\begin{array}{l}\text { Input signal for controlled element } \\
\text { (rad). }\end{array}$ \\
\hline $\begin{array}{l}F_{\text {dist }} \\
F_{\text {hapt }}\end{array}$ & $\begin{array}{l}\text { Disturbance force }(\mathrm{N}) \text {. } \\
\text { Force generated by haptic system }(\mathrm{N}) \text {. }\end{array}$ & $x$ & $\begin{array}{l}\text { Position of the controlled element } \\
\text { (rad). }\end{array}$ \\
\hline$F_{\text {hum }}$ & Total human force $(\mathrm{N})$. & $x_{C D}$ & Control device deflection (rad). \\
\hline$F_{l i n}$ & Pilot linear response $(\mathrm{N})$. & $x_{N P S}$ & Neutral point shift (rad). \\
\hline$F_{v i s}$ & Human force given by visual system & $x_{t a r}$ & Target position (rad). \\
\hline
\end{tabular}

\section{Introduction}

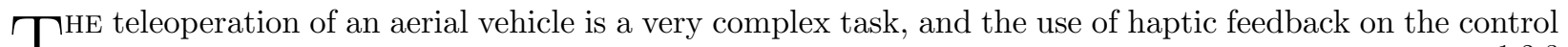
1 stick for complementing the visual information has been shown to improve the pilot performance $1 \frac{12 \sqrt{2}}{3}$ Pilots use the haptic aid to adapt their control behaviour in order to increase their performance.

It is a common understanding that admittance, which is defined as the causal dynamic relationship between the force acting on the limb (input) and the position of the limb (output), could play an important role: humans are able to modify their admittance in relation to the task they perform. Most experimental studies concentrated on neuromuscular adaptive behaviour in the so called "classical tasks" (position, force and relax task) $4[5] 6] 7[8] 9$ These studies did not investigate the changes in pilot admittance during more complex tasks; furthermore the visual response was considered irrelevant.

Other studies showed the variability of admittance in a pitch control task with visual feedback 10,11 Recently the theories about the adaptability of admittance with the task were applied to a car driver with haptic aid 12 The authors estimated the admittance of the ankle-foot complex while performing a carfollowing task, separating the driver's responses to visual feedback from those in response to designed force disturbance. In this case, a Direct approach (DHA) was used for the design of the haptic aid; in this experiment, the pilot's neuromuscular system had to be compliant with the force felt on the gas pedal to benefit from the haptic aid and achieve the task. A different haptic approach is the Indirect Haptic Aiding (IHA) 13 that helps the pilots indirectly by letting them know through the sense of touch what is happening in the environment. IHA tries to exploit pilot's natural counteracting responses to what he perceives as a disturbance. In general, this haptic aid provides a force that has the opposite sign with respect to the one required in order to achieve the task; the operator has to oppose to the haptic force to yield adequate performance.

Various pilot models were developed that describe the characteristics of the human operator in response to visual stimuli in several tasks (compensatory task, pursuit task, with or without motion cues) $14 \sqrt[15]{15}$ Many different model structures were derived to represent the pilot's response to visual errors and perceived accelerations, such as the Crossover Model, the Extended Crossover Model, the Precision Model ${ }^{16}$ These models usually do not distinguish between the human pilot and the control inceptor: these are lumped together and there is no indication about the pilot's control strategy in response to the haptic feedback.

In order to include the contribution of the haptic aid, the neuromuscular system model needs to be separated from the control device model. Multi-loop models can be used for this purpose. Separating the neuromuscular system model from the control device allows supplementing the pilot model with measure- 
ments for arm admittance. This type of models was already applied in different scenarios, like pitch tracking task with different side stick configuration ${ }^{11}$ and car-following task with DHA. ${ }^{12}$

This paper presents a multi-loop model capable of describing the pilot behaviour during a pitch tracking task with different haptic aids. The goal is to better understand how the pilot adapts the neuromuscular and visual response in relation to different types of haptic cues. In addition, the pilot open-loop transfer function is analysed and compared with the well-documented behaviour without haptic aids.

Multiloop identification methods are needed in order to estimate both the arm admittance and the visual response. The technique used in previous works $\frac{11}{12}$ is based on cross-spectral density estimates and allows the determination of the desired transfer functions, but assumes some hypotheses which are not always fulfilled. Two different approaches which do not require the same assumptions, are investigated in this paper. The first is based on polynomial linear time invariant systems, in particular Auto Regressive with eXogenous input models (ARX). The second one is still based on cross-spectral density estimates, but adopts a different procedure to compute the pilot admittance.

The paper is structured as follows: first we introduce and discuss a pilot model that is suitable for the purposes of this paper; then, the theoretical basis of the three adopted identification methods are illustrated, followed by a set of Monte Carlo simulations performed in order to investigate their performances. Furthermore, an overview of the different haptic aids used in the experiment is presented. Subsequently, the experimental setup is discussed and the results are given. Finally, conclusions are drawn.

\section{Multichannel pilot model}

The human operator is a nonlinear biological system, but it is usually approximated it with a quasi-linear time-invariant model with a remnant signal that describes the nonlinear behaviour. During manual-control tasks, human operators uses information perceived through different perceptual channels; in this paper, pilot control behaviour during compensatory pitch control tasks is investigated. The operator is presented with a display of tracking error alone. No informations about target signal $x_{t a r}$ and system position $x$ are shown to the pilot, but only the error $e$ between them.

Several pilot models have been extensively used in the study of human control behaviour during a compensatory tracking task $17 \sqrt[16]{15}$ In those models (figure 1), the arm's neuromuscular system (NMS) and the control device $(\mathrm{CD})$ are lumped together.

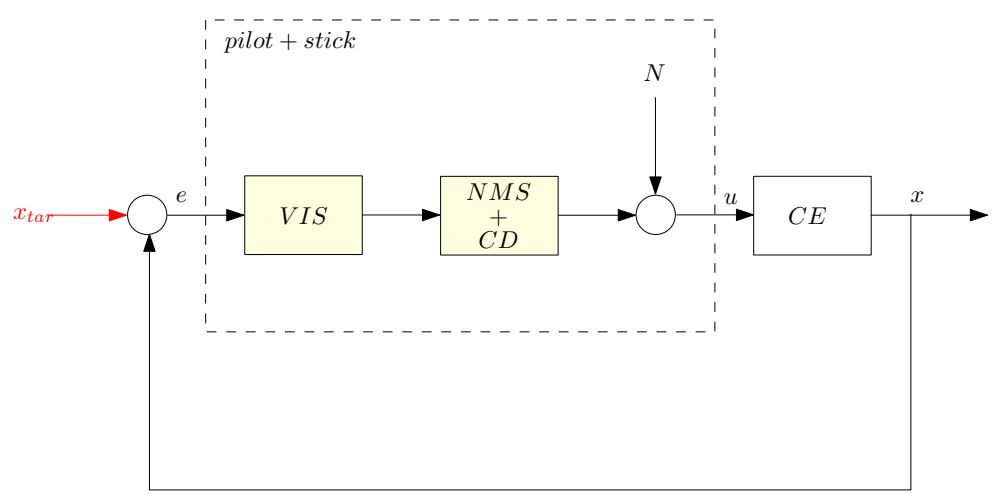

Figure 1. Pilot pitch-tracking control loop with neuromuscular system (NMS) and control device (CD) lumped together.

The visual error $e$ is the only input for the operator and it generates the command signal $u$ for the controlled element $(C E)$ passing through the visual system (VIS) and the lumped block NMS+CD. An additional remnant signal $N$ is added to the pilot linear response to model nonlinearities. McRuer $\frac{17}{17}$ theories assess that the frequency response function (FRF) of the pilot $H_{p}$, which includes both pilot NMS and stick dynamics, combined with the FRF of the controlled element $H_{C E}$ approximate the dynamics of a single integrator around the crossover frequency $\omega_{c}$ :

$$
H_{p}(j \omega)=\frac{\omega_{c}}{j \omega} e^{-j \omega \tau_{e}}
$$

where the equivalent pilot time delay $\tau_{e}$ accounts for phase lag around crossover frequencies.

When haptic aid systems are involved, the pilot receives additional information on a possible control strategy. It is not known how the pilot adapts his behaviour to different haptic aids and whether McRuer 
theories are still valid when haptic aids are applied. Pilots can feel the haptic aid through the control device, therefore the haptic input, which may be an additional force on the stick or a variation of its characteristics, must be considered as another input for the human operator. This could be modelled with a multiloop (or multichannel) model. Multichannel models have already been used to describe the pilot behaviour in different experimental setup, such tracking with simulator motion $\sqrt{18}[19$ In order to describe the influence of the haptic feedback on the pilot, the control inceptor needs to be separated from the neuromuscular system. A pilot model in which the stick and the pilot are not lumped together is shown in figure 2 .

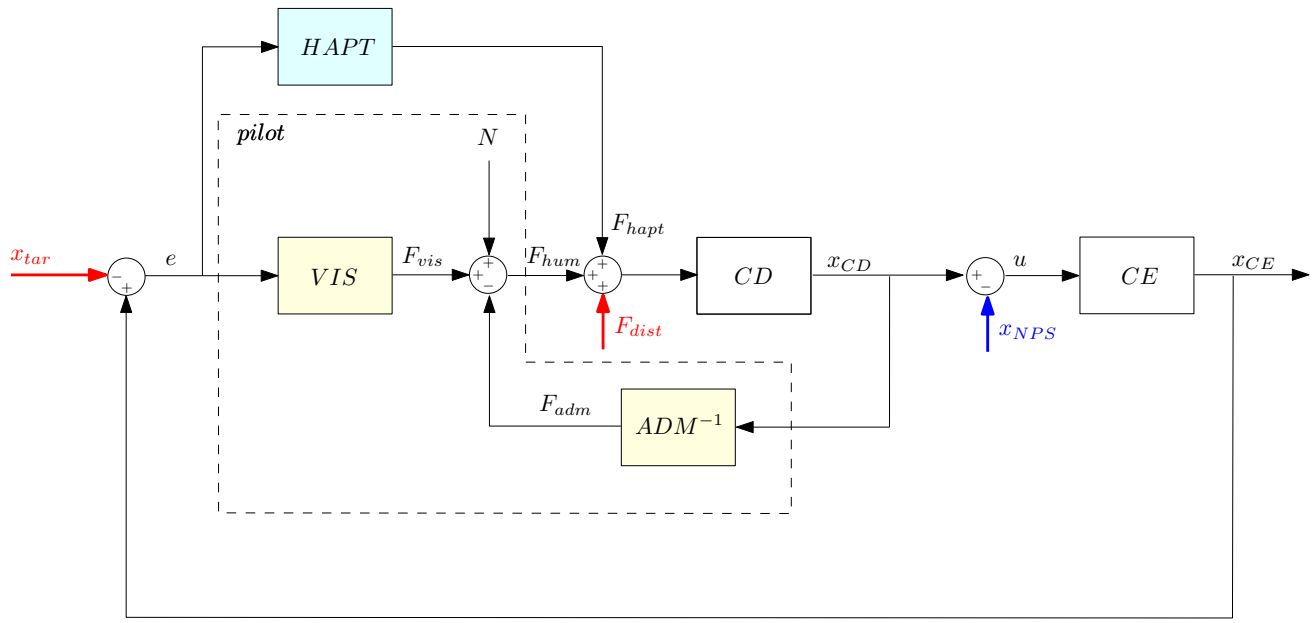

Figure 2. Pilot pitch-tracking control loop, including haptic aid. The neuromuscular system is described by the admittance (ADM) and is modelled separately from the control device (CD).

The pilot is described by a two inputs and single output system. Pilot control action $F_{\text {hum }}$ is shown to be the sum of three different contributions: the pilot's visual response $F_{v i s}$, produced by the visual system VIS with visual error $e$ as input, the neuromuscular response $F_{a d m}$ to the motion of the stick $x_{C D}$, and the remnant $N$. The neuromuscular system is described by the inverse of the arm admittance ADM, which is defined as the causal dynamic relationship between the force $F_{A D M}$ acting on the arm (input) and the position of the arm $x_{C D}$ (output). The haptic aid is represented by the HAPT block, and generates forces depending on the dynamics of the visual error $e$. Various haptic systems will be described in section $\mathrm{V}$. Similar models were used to describe pilot pitch-tracking task without haptic feedback 20 The same scheme was used to model the leg car drivers during a car-following task with haptic aid ${ }^{21}$ The signal $x_{N P S}$ represents an optional neutral point shift (NPS); this will be used to implement the IHA in section V

\section{Identification methods}

It is well known in literature that humans are able to adapt their behaviour in relation to the so called classical task they perform $4 \sqrt[422]{ }$ Classical tasks require the human to minimize the stick deviation by resisting stick forces (position task, PT), to stay totally relaxed in face of continuous stick forces (relax task, RT) and to minimize forces applied to the stick (force task, FT). As described in previous studies, ${ }^{21}$ humans are able to change their neuromuscular settings also during a tracking task, for example depending on whether or not there is haptic aid. Only a direct haptic approach was investigated in that work (refer to section $\mathrm{V}$ for further information).

This paper investigates how pilots adapt neuromuscular and visual settings in relation of different types of haptic aid, and how the pilot open loop transfer function varies by using haptic cues. Identification techniques are needed to obtain both neuromuscular and visual systems estimates for a multi-loop pilot model with neuromuscular system separated from control device (figure 2).

This section presents an overview of the techniques that will be used and compared against in this paper.

\section{III.A. Identification for classical tasks}

During classical tasks the contribution of visual system can be considered negligible $\left(F_{v i s}=0\right)$ as the task instruction is purely force related, and the humans can be described by a single-input and single output 
closed loop system. The model in figure 2 can be rearranged as in figure 3

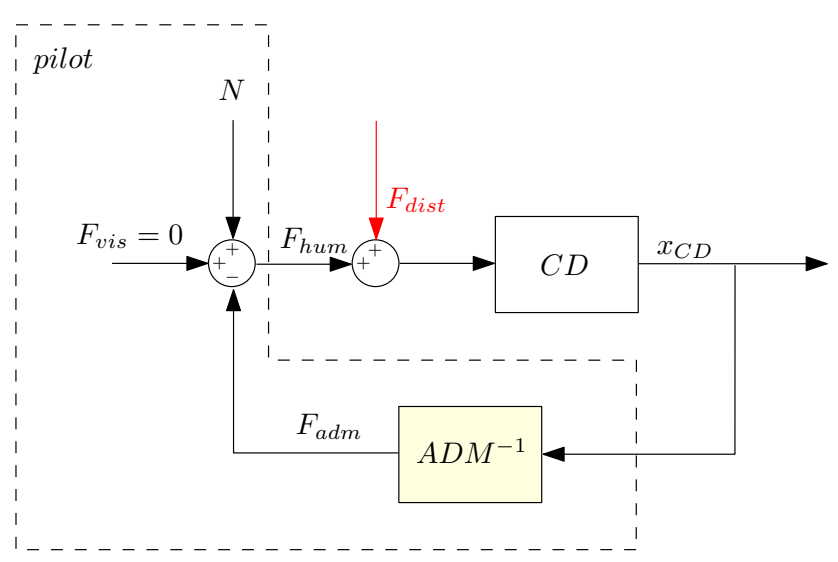

Figure 3. Human control loop for a classical task. The neuromuscular admittance (ADM) and the control device (CD) form a closed-loop system. The visual response $F_{V I S}$ can be considered negligible. The remnant $N$ models pilot nonlinearities.

Considering this model, well-known techniques are available for identification of the human admittance $7[5$

The signal $F_{\text {dist }}$ represents the disturbance forcing function, that is a force applied to the CD for identification purposes only. $F_{\text {dist }}$ was designed as a multisine generated offline in the frequency domain at a set of frequencies labelled set $f_{d} \cdot 9$ The phase was randomized to yield an unpredictable disturbance signal, and a cresting technique was applied to avoid peaks in the time domain $23[9]$ The signal amplitudes was generated using "Reduced Power Technique", 24 that enables admittance estimation for a large range of frequencies without influencing low-frequency behaviour. Figure 4 shows the power spectral density (PSD) and time realization of the force disturbance. The values of PSD at low frequencies (FP) are higher than the values at high frequencies (RP), such that the higher frequencies does not influence the control behaviour but still allow estimation at high frequencies.

The relationship between the human force $F_{\text {hum }}$ (input) and the stick deflection $x_{C D}$ (output), i.e. the arm admittance, can be estimated as:

$$
\hat{H}_{a d m}\left(f_{d}\right)=\frac{\hat{S}_{F_{\text {dist }} x_{C D}}\left(f_{d}\right)}{\hat{S}_{F_{\text {dist }} F_{\text {hum }}}\left(f_{d}\right)}
$$

The term $\hat{S}_{F_{d i s t} x_{C D}}\left(f_{d}\right)$ is the estimate of the cross-spectral density of disturbance force $F_{d i s t}$ and stick deflection $x_{C D}$, whereas the term $\hat{S}_{F_{\text {dist }} F_{\text {hum }}}\left(f_{d}\right)$ is the estimate for the cross-spectral density of disturbance force and human force $F_{\text {hum }}$.

The squared coherence function $\hat{\Gamma}^{2}(\cdot)$ can be used as a measure of signal to noise ratio and linearity of the admittance estimate:

$$
\hat{\Gamma}^{2}\left(f_{d}\right)=\frac{\left|\hat{S}_{F_{d i s t} x_{C D}}\left(f_{d}\right)\right|^{2}}{\hat{S}_{F_{\text {dist }} F_{\text {dist }}\left(f_{d}\right) \hat{S}_{x_{C D} x_{C D}}\left(f_{d}\right)}}
$$

The coherence can be 1.0 if the system under study is actually linear and decreases toward 0.0 with noise and unmodelled nonlinearities.

\section{III.B. Identification for the tracking task}

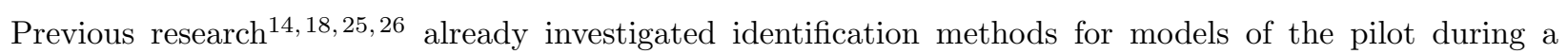
tracking task. These works considered stick and neuromuscular dynamics lumped together. The estimation becomes more involved when the pilot is modelled with separated CD and neuromuscular system, as in figure 2. This approach was used for a car-driver in a car-following task, 21 and for a pilot in a pitch tracking task ${ }^{11}$ The identification method used was based on cross-spectral density estimates. This technique is capable of measuring neuromuscular behaviour while performing a vehicular control task, but requires the assumption that the forcing forces used for admittance identification do not appear in the visual feedback error; this assumption, as it will be described and discussed later, is difficult to fulfil in a tracking task.

In this paper, two techniques different from the well known approach will be developed in order to manage the case in which the hypothesis is not fulfilled. In the first one, polynomial models are used to describe the 
dynamics of the measured data. This technique was recently applied with success for the identification of pilot models with motion cues ${ }^{18}$ and can be extended to the case with haptic aid. An ARX model is fitted to the measured data and the pilot admittance and other response functions are calculated analytically from this estimated model. The second one has not been applied before for the pilot admittance and visual system identification. In this method, two different FRFs are estimated using cross-spectral density estimates and the desired admittance and visual FRFs are obtained using an interpolation procedure and diagram block algebra.

\section{Disturbance signal design}

The design of the disturbance signals was chosen in the same way for all the presented identification methods. Two different signals, i.e. a target and a disturbance forcing function, are needed to allow for separate modelling of pilot visual response and arm admittance. As shown in figure 2, the target signal $x_{\text {tar }}$ represents the reference trajectory that needs to be followed, whereas the disturbance signal $F_{\text {dist }}$ represents the additional force in the stick for identification purposes.

Simultaneously applying two forcing signals requires a method that allows to distinguish the contribution from each signal in the measurements. A well documented method ${ }^{12} 27$ consists of separation of the signals in the frequency domain. Different sets of discrete points in the available frequencies range were assigned to the two signals, in order to distinguish the response to each signal during the analysis. In this paper, each set of frequency points was composed by two adjacent frequency points to allow for frequency averaging. The first group of two frequency points was assigned to $F_{d i s t}$, the second group was assigned to $x_{t a r}$, the third and the fourth group did not contain any power, the following group was again assigned to $F_{d i s t}$, and so on. Figure 4 shows the frequency separation of the two forcing signals.

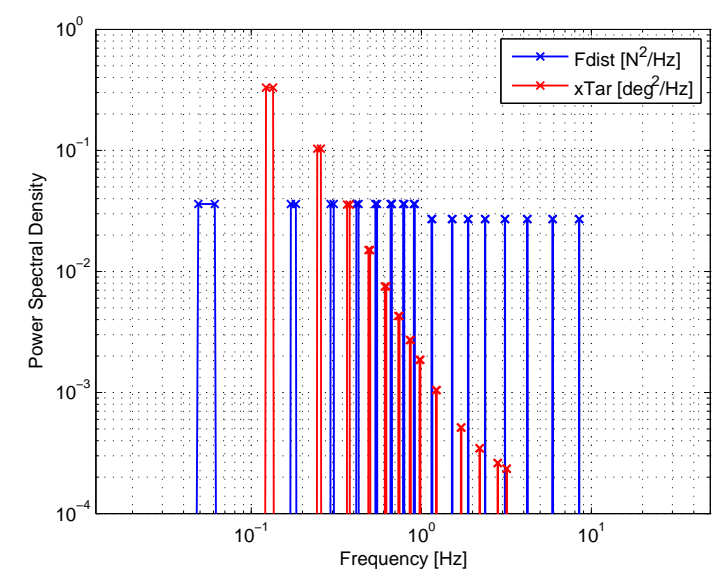

(a) Frequency domain.
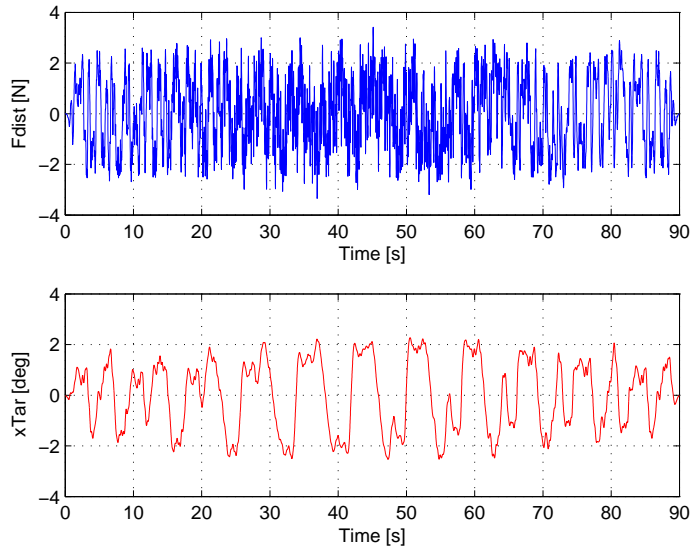

(b) Time domain.

Figure 4. Power spectral densities (left) and time realization (right) of forcing signals $x_{t a r}$ and $F_{d i s t}$.

For the target position $x_{t a r}$, a range between 0.001 and $3 H z$ was selected. The distribution of amplitudes was chosen with the following filter 18

$$
H_{f}=\frac{(s+10)^{2}}{(s+1.25)^{2}}
$$

Random phases were chosen to obtain unpredictable behaviour and a cresting technique was applied to avoid amplitude peaks in time realization.

The force disturbance $F_{\text {dist }}$ was similar to that used in classical tasks. In accordance with the 'Reduced Power Technique',24 it contained full power up to $1 \mathrm{~Hz}$ and reduced power up to $10 \mathrm{~Hz}$. The full power gain FP and the reduced power gain RP were calibrated to reach a trade-off between good signal-to-noise ratio in the measured signals and low influence on the human admittance. The FP and RP gains were minimized heuristically via tuning experiments aimed at minimizing the influence of the disturbance on the human operator behaviour and on the estimated admittance, while providing the highest possible coherence function. 
The multisine time signals were obtained using the inverse Fast Fourier Transform ${ }^{9}$ Figure 4 shows the time realisations of $F_{d i s t}$ and $x_{t a r}$, cut to a length of $90 \mathrm{~s}$. Both signals were smoothed at the beginning and at the end of the time interval, in order to avoid transient effect. In the experiment, data were logged at 100 $\mathrm{Hz}$, resulting in 9000 samples. Only $8192\left(2^{13}\right)$ were used for data analysis.

\section{Cross-spectral densities}

A possible approach for identification of admittance and visual response uses cross-spectral density estimates (CSD). In the rest of the paper, we will refer to this technique as CSD-based method. This approach has been previously used for pilot estimation in a pitch tracking task 10 and for car driver estimation in a car following task 12

In a closed multi-loop system like in figure 2, the pilot admittance can be calculated at frequencies $f_{d}$ using the same procedures used for classical tasks:

$$
\hat{H}_{a d m}\left(f_{d}\right)=\frac{\hat{S}_{F_{\text {dist } x C D}}\left(f_{d}\right)}{\hat{S}_{F_{d i s t} F_{a d m}}\left(f_{d}\right)}
$$

where $\hat{S}_{F_{\text {dist }} x_{C D}}\left(f_{d}\right)$ and $\hat{S}_{F_{d i s t} F_{a d m}}\left(f_{d}\right)$ are the estimated cross-spectral density between $F_{d i s t}$ and $x_{C D}$ and between $F_{d i s t}$ and $F_{a d m}$ Usually, it is not possible to measure $F_{a d m}$, but only the total human force $F_{\text {hum }}$. If the visual response $F_{V I S}$ does not contain power at frequencies $f_{d}$, then only $F_{a d m}$ significantly contributes to the human force $F_{\text {hum }}$ measured at $f_{d}$ frequencies. Therefore, if the power content of the tracking error $e$ is almost zero for $f_{d}$ frequencies, it is possible to estimate the admittance as in Eq. (2). Clearly this procedure estimates the admittance assuming linearity of the model. The squared coherence function in Eq. (3) was used to check the validity of this hypothesis.

The pilot visual response $H_{V I S}$, i.e. the FRF of the block VIS in figure 2, can not obtained directly with the CSD-based method. On the contrary, the pilot open-loop transfer function, defined as the transfer function from the visual error $e$ to the position of the controlled device $x_{C D}$, can be estimated in the frequency domain using cross-spectral density estimates. The pilot open-loop FRF at frequencies $f_{t}$ can be estimated as: 25

$$
\hat{H}_{O L}\left(f_{t}\right)=\frac{\hat{S}_{x_{\text {tar } x}\left(f_{t}\right)}}{\hat{S}_{x_{\text {tar }}}\left(f_{t}\right)}
$$

Also for the open-loop FRF, the coherence function was used as a measure for the reliability of the model:

$$
\hat{\Gamma}_{O L}^{2}\left(f_{t}\right)=\frac{\left|\hat{S}_{x_{\text {tar }} x}\left(f_{t}\right)\right|^{2}}{\hat{S}_{x_{\text {tar }} x_{\text {tar }}}\left(f_{t}\right) \hat{S}_{x x}\left(f_{t}\right)}
$$

Summarizing, this method assumes that the visual loop can be cut for admittance estimation, because the tracking error has no power at frequencies $f_{d}$. If this hypothesis is not fulfilled, this method could give unreliable estimates. Two different identification methods were developed. In these methods, the admittance and visual response are estimated simultaneously, without assuming a complete frequency separation between the disturbance force $F_{d i s t}$ and the visual error $e$.

\section{Linear Time-Invariant models}

The identification of the admittance and visual response can be performed also using linear time-invariant models (such ARX, ARMAX, OE, BJ). Consider the multi-input pilot model in figure 2. In this case the CD deflection $x_{C D}$ can be written as the sum of three contributions, one caused by the visual error, one by the force disturbance, and one by noise:

$$
x_{C D}=H_{1} e+H_{2} F_{d i s t}+H_{N} N
$$

where $H_{1}, H_{2}$, and $H_{N}$ are the transfer functions respectively from $e, F_{d i s t}$, and $N$ to $x_{C D}$.

This system has the same structure of multi-input-single-output (MISO) polynomial linear models. 28 Therefore, given the measurement of $x_{C D}, e$ and $F_{d i s t}$, a prediction error method can be used to fit the data with a polynomial model. In this work, ARX models were used to fit the data:

$$
A(q) y(t)=B_{1}(q) u_{1}(t)+B_{2}(q) u_{2}(t)+n(t)
$$

$$
7 \text { of } 23
$$


With ARX models, the polynomial orders and the parameter values can be easily found as the optimal solution of a proper cost function (like the Akaike final prediction error) ${ }^{28}$ In order to avoid overfitting of the parameters, the measurements were divided in two parts in the time domain. The first one was used for model identification, the second one for model validation. The disadvantage of the ARX models is that they use a predefined model structure in which all the transfer functions of the system have the same set of poles. The coupling between the deterministic and the stochastic part of the system can be unrealistic.

The frequency function values of the arm admittance, visual response and open loop transfer function can be easily obtained from the transfer functions $H_{1}$ and $H_{2}$ using block diagram algebra:

$$
\begin{aligned}
H_{a d m} & =\frac{H_{C D} H_{2}}{H_{C D}-H_{2}} \\
H_{v i s} & =\frac{H_{1}+H_{\text {hapt }} H_{2}}{H_{2}} \\
H_{O L} & =H_{1} H_{C E}
\end{aligned}
$$

where $H_{k}$ represents the FRF of the block $k$.

An overall validity index for the ARX models is obtained by the Variance Accounted For (VAF), which gives informations about the capability of the model to repeat the measured output signals given the same inputs. The VAF is defined as:

$$
V A F=\left[1-\frac{\sum_{k=1}^{N}\left|x_{C D}\left(t_{k}\right)-\hat{x}_{C D, A R X}\left(t_{k}\right)\right|^{2}}{\sum_{k=1}^{N}\left|x_{C D}\left(t_{k}\right)\right|^{2}}\right] \times 100 \%
$$

where $x_{C D}\left(t_{k}\right)$ and $\hat{x}_{C D, A R X}\left(t_{k}\right)$ represent respectively the measured and the simulated stick deflection (k indexes the time samples). A VAF of $100 \%$ indicates that the ARX model completely describes the measured system response, whereas lower values are an indication of a worse model fit due to noise, nonlinearities, or other unmodelled system characteristics.

In summary, the ARX method has the disadvantage of using a predefined model structure (linear ARX model), but the advantage of not requiring any assumptions on the power spectrum of the visual error.

\section{Multi-loop cross-spectral densities}

In order to avoid rigid assumptions about the model structure, a third identification method was developed. This method uses cross-spectral density estimates as the CSD-based method, but it does not require any hypotheses about the power content of the visual error $e$. In the rest of the paper we will describe this method as "CSD-ML method".

Consider the pilot model shown in figure 2. The FRFs from the two forcing functions $x_{t a r}$ and $F_{d i s t}$ to the CD deflection $x_{C D}$ can be estimated using cross-spectral density estimates $29 \mid 23$

$$
\begin{gathered}
\hat{H}_{t a r}\left(f_{t}\right)=\frac{\hat{S}_{F_{\text {tar } x} x_{C D}}\left(f_{t}\right)}{\hat{S}_{F_{\text {tar }} F_{t a r}}\left(f_{t}\right)} \\
\hat{H}_{\text {dist }}\left(f_{d}\right)=\frac{\hat{S}_{F_{\text {dist } x} x_{C D}}\left(f_{d}\right)}{\hat{S}_{F_{\text {dist }} F_{\text {dist }}\left(f_{d}\right)}}
\end{gathered}
$$

Since the two estimates of $\hat{H}_{t a r}$ and $\hat{H}_{d i s t}$ are obtained for different sets, i.e. $f_{t}$ and $f_{d}$, a linear interpolation between neighbouring frequencies was used, assuming "smooth" transfer functions, to extrapolate estimates of both FRF for all the frequency points $f_{d}$ and $f_{t}$. It should be noted that, considering the disturbance forcing functions designed as in section III.B, $f_{d}$ ranges from $0.01 \mathrm{~Hz}$ to $10 \mathrm{~Hz}$, whereas $f_{t}$ contains only frequencies below $3 \mathrm{~Hz}$. Thus any extrapolated value of $\hat{H}_{\text {tar }}$ above $3 \mathrm{~Hz}$ can not be considered reliable. This means that with the CSD-ML method we could reliably estimate the admittance only at frequencies below $3 \mathrm{~Hz}$. Furthermore, the admittance estimate can not considered accurate even at the frequency $f_{d_{1}}$, that is the minimum frequency point of $f_{d}$. In fact, as $f_{d_{1}}$ is lower than the minimum frequency point of $f_{t}$, the estimation of $\hat{H}_{t a r}\left(f_{d_{1}}\right)$ is obtained extrapolating the values of $\hat{H}_{t a r}\left(f_{t}\right)$. It is likely that the extrapolation does not give reliable values as linear interpolation between neighbouring frequencies. 
The FRFs of pilot admittance, visual response and open-loop transfer function can then be estimated using diagram block algebra:

$$
\begin{aligned}
\hat{H}_{a d m}(\tilde{f}) & =\frac{\hat{H}_{\text {dist }}(\tilde{f}) H_{C D}}{H_{C D}-H_{C D} \hat{H}_{\text {tar }}(\tilde{f}) H_{C E}-\hat{H}_{\text {dist }}(\tilde{f})} \\
\hat{H}_{\text {vis }}(\tilde{f}) & =-\frac{\hat{H}_{\text {tar }}(\tilde{f})}{\hat{H}_{\text {dist }}(\tilde{f})}-H_{\text {hapt }} \\
\hat{H}_{O L}(\tilde{f}) & =-H_{C E} \frac{\hat{H}_{\text {tar }}(\tilde{f})}{1-\hat{H}_{t a r}(\tilde{f}) H_{C E}}
\end{aligned}
$$

This technique does not assume any predefined model structure and does not make any hypothesis about the power content of the visual error $e$. The disadvantage, at least in our case, is that it gives reliable estimates only for low frequencies.

\section{Off-line simulations}

This section presents a comparison and validation of the three identification techniques presented in section III.B. A Monte-Carlo simulation using a model of the pilot's visual response and admittance was used for validation. The model presented in figure 2 was simulated with 100 different realizations of the remnant noise, in order to investigate the robustness of the three identification methods. The remnant noise was obtained as Gaussian white noise $n_{w}$ filtered by a third-order-low-pass filter ${ }^{26}$

$$
H_{n}(j \omega)=K_{n} \frac{\omega_{n}^{3}}{\left(j \omega+\omega_{n}\right)\left((j \omega)^{2}+2 \xi_{n} \omega_{n} j \omega+\omega_{n}^{2}\right)}
$$

where $\xi_{n}=0.26$ and $\omega_{n}=12.7 \mathrm{rad} / \mathrm{s}$ are the remnant-filter damping factor and break frequency, respectively. The gain $K_{n}=2.12$ was selected in order to obtain the ratio 0.5 between the power of the linear pilot response $F_{\text {lin }}\left(F_{\text {lin }}=F_{v i s}-F_{a d m}\right)$ and the remnant power. Figure 5 shows typical time realisations of $F_{\text {lin }}$ and $N$ for one simulation.

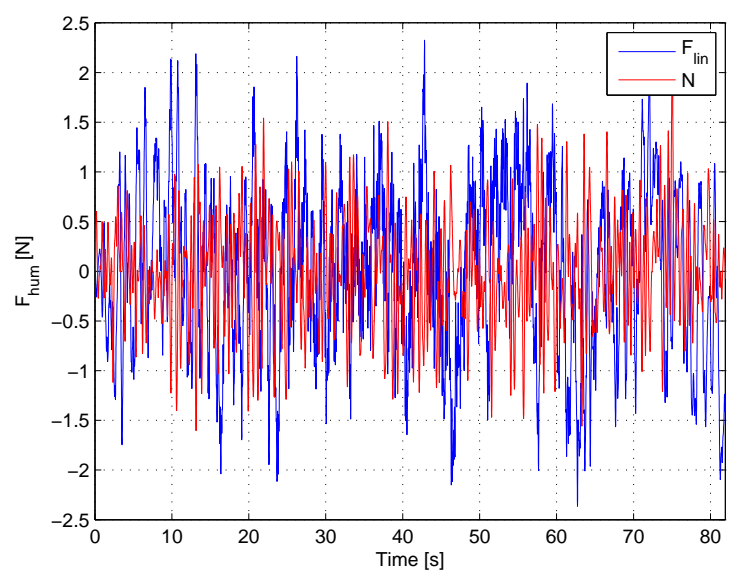

Figure 5. Time domain representations of the linear pilot response $F_{l i n}$, and remnant $N$. by

Each simulation used the same parameters for all the blocks in the model. The CE dynamics was modelled

$$
H_{C E}=6 \frac{3}{s(s+3)} \quad[\mathrm{rad} / \mathrm{rad}]
$$

The control device CD was described by the identified model of the real stick used during the experiment (section VI.B):

$$
H_{C D}(s)=\frac{1}{1.522 s^{2}+8.832 s+86.469} \quad[\mathrm{rad} / \mathrm{N}]
$$


The values of the parameters of the pilot admittance and visual response were derived from previous work, 11 in which a non-parametric estimate of the admittance, and a parametric estimate of the visual response, were obtained for pilots in a pitch tracking task. The arm admittance was modelled by:

$$
H_{a d m}=\frac{4.566 \cdot 10^{-6}(j \omega)^{3}+0.0046(j \omega)^{2}+1.333(j \omega)+97.52}{(j \omega)^{3}+82(j \omega)^{2}+712.2(j \omega)+1.167 \cdot 10^{-4}} \quad[\mathrm{rad} / \mathrm{N}]
$$

The resulting admittance is similar to that we found successively in our experiments. The model of the visual response was given by a gain, lead-lag filter for equalization and a time delay:

$$
H_{v i s}(j \omega)=K_{v} \frac{1+j \omega T_{l e a d}}{1+j \omega T_{l a g}} e^{-j \omega \tau_{v}} \quad[N / \mathrm{rad}]
$$

where $K_{v}=20 \mathrm{~N} / \mathrm{rad}, T_{l e a d}=0.3 \mathrm{~s}, T_{\text {lag }}=0.04 \mathrm{~s}$, and $\tau_{v}=0.2701 \mathrm{~s}$. The parameter values were adapted from those in the cited reference so that the time domain realizations of the simulated signals were more similar to those measured during the experiment (section VII). No haptic aid was used during the simulations, thus $H_{\text {hapt }}=0$.

The design of the forcing functions $F_{d i s t}$ and $x_{t a r}$ was largely discussed in section III.B. The two input signals $F_{d i s t}$ and $x_{t a r}$ were chosen as multisine signals generated off-line and separated in the frequency domain.

The CSD-based, ARX, and CSD-ML identification methods were applied to the output of the 100 simulations with different noise realizations. Each simulation lasted 81.92s. A typical power spectral density PSD of the visual error $e$ is given in figure 6 .

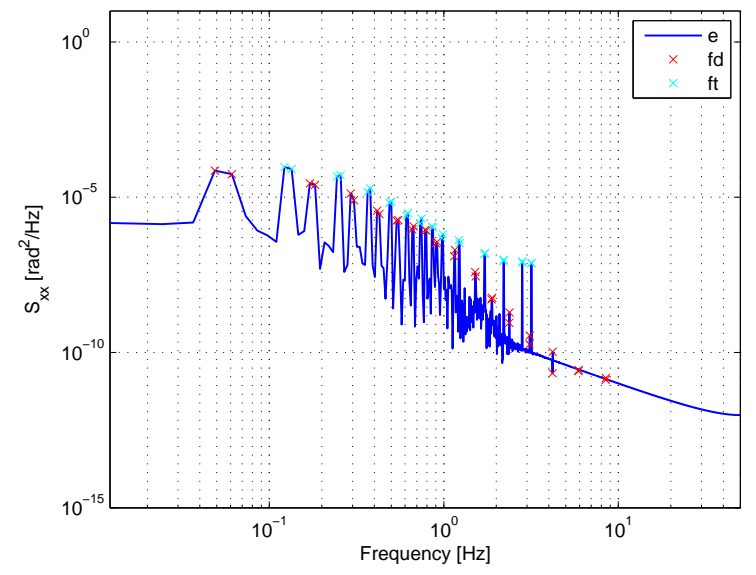

Figure 6. PSD of the visual error $e$. The crosses represent the disturbance force and target position frequencies.

The contributions of each forcing function are easily distinguishable. The cyan crosses indicate the frequencies where the target position $x_{t a r}$ had power and the red crosses is where the force disturbance $F_{d i s t}$ had power. The force disturbance $F_{\text {dist }}$ has a large influence on the visual error $e$, thus the hypothesis of the CSD-based identification method is not fulfilled. Therefore, the latter two methods, ARX and CSD-ML, should estimate the frequency response of the admittance better than the CSD-based approach.

The admittance estimates given by the three identification methods are shown in figure 7 As expected, the CSD-based approach does not give correct estimates of the admittance at low frequencies. The power content of $e$ is not negligible at frequencies $f_{t}$ and thus influences the admittance estimation in the CSDbased method, leading to unreliable estimates. On the contrary, both ARX and CSD-ML methods give good results. As expected and motivated in section III.B the CSD-ML does not give reliable admittance estimate at the minimum frequency value of $f_{d}$. The same issue will be observed with the experimental data.

The pilot visual response can be estimated only with ARX and CSD-ML approaches; figure 8 shows that both methods give reliable estimates.

Finally, the estimated open-loop transfer functions well fit the simulated one for all the three identification methods (figure 9). 


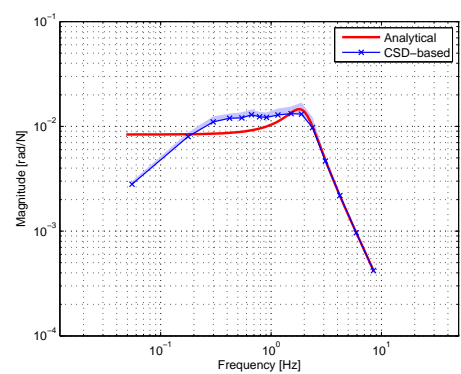

(a) CSD-based.

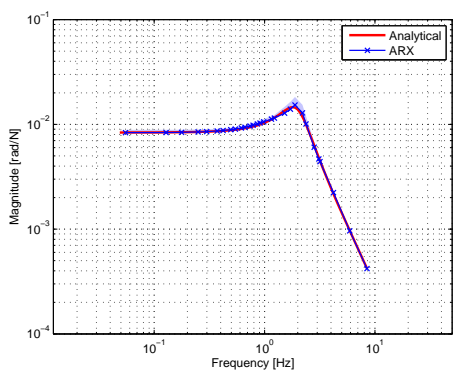

(b) ARX.

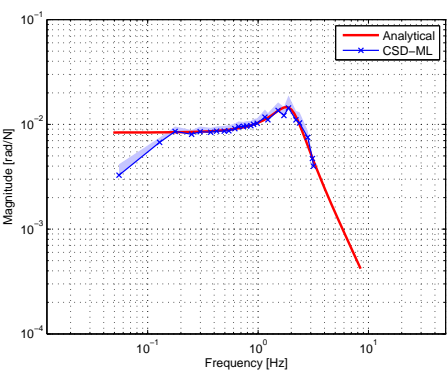

(c) CSD-ML.

Figure 7. Comparison between admittance estimates given by the three identification methods. The estimates are averaged over all the simulations, and the shaded areas show the standard deviation.

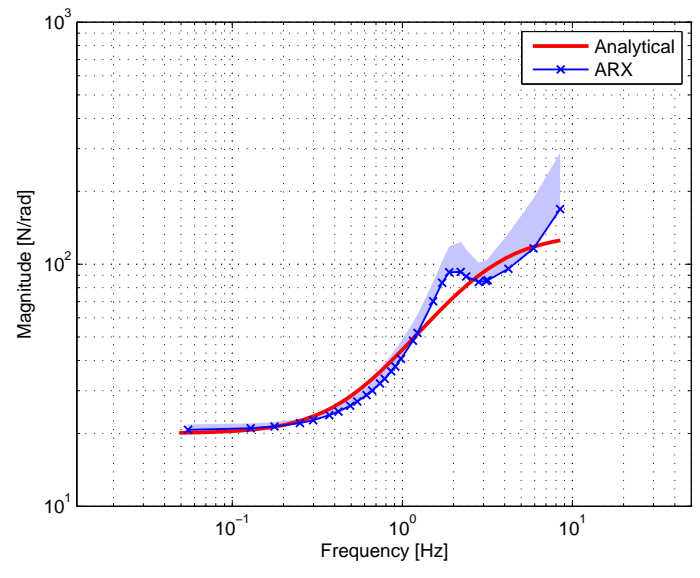

(a) ARX.

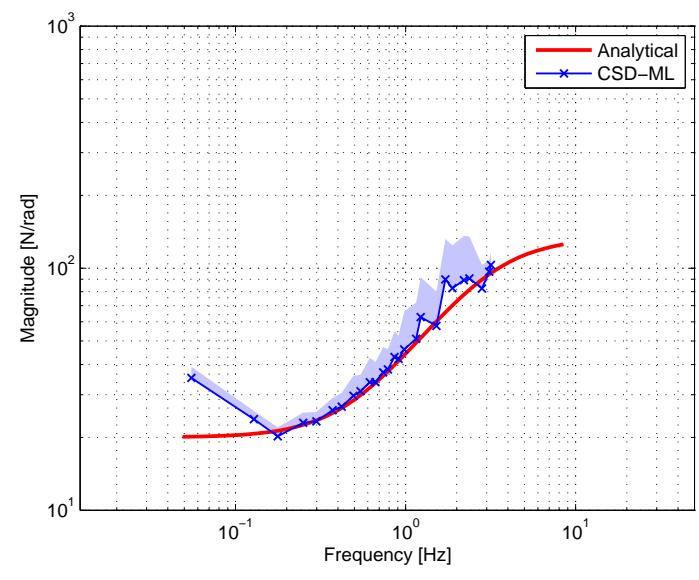

(b) CSD-ML.

Figure 8. Comparison between visual responses given by ARX and CSD-ML methods.

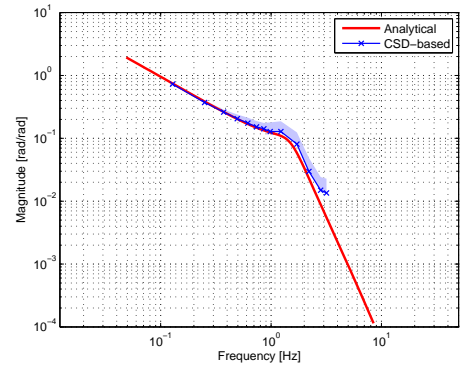

(a) CSD-based.

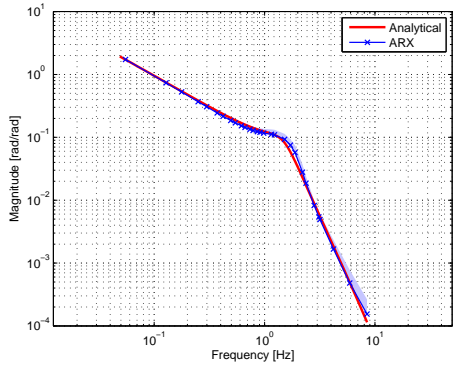

(b) ARX.

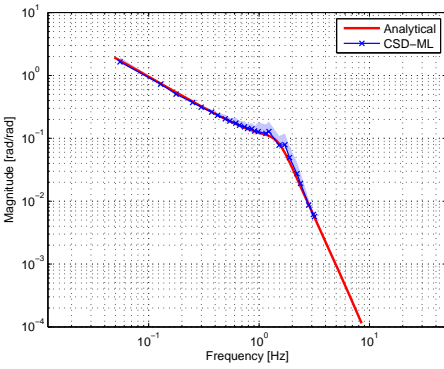

(c) CSD-ML.

Figure 9. Comparison between open-loop transfer function estimates given by the three identification methods. 


\section{Haptic aids}

Haptic aids refer to systems that provide touch sensations to their operators, in addition to conventional visual informations. Haptic technology has been used in many fields, like telerobotic controls, $\stackrel{31}{r}$ virtual reality $\underline{32}$ and so on. Several applications of haptic cues in aerial vehicles operations have been analysed in literature, e.g. a vibrating wristband was used to alert pilot about dangerous situations. ${ }^{33}$

Haptic information can be provided to vehicle pilots through an active control device: the haptic system applies to the stick external forces or sensations (due to stick stiffness changes for instance) in order to make the task easier for pilots. This kind of haptic aids can be divided in two philosophies:13

- Direct Haptic Aid (DHA): the class of those haptic aids that produce forces in order to help the pilot suggesting the right control action. The operator has to be compliant with the force in order to achieve the task.

- Indirect Haptic Aid (IHA): the class of those haptic aids that aim at increasing the pilot situational awareness (e.g. of the existence of a tracking error) leaving him full control authority. The operator has, in general, to oppose to it in order to achieve the task.

It is clear that these two class of haptic aids are complementary and in most cases an IHA system may be derived from a corresponding DHA by inverting the sign of the feedback force and shifting of the neutral point of the control device.

DHA approach has been largely applied in previous works. A direct haptic aid was used to support the car driver during a car following task ${ }^{21}$ Several studies investigated the use of force-stiffness feedback, i.e. a combination of force offset and extra spring load, in UAV teleoperation for obstacle avoidance $\sqrt[34 \sqrt[35]{3 H A}]{\text { IHA }}$ approach was applied to wind rejection in remotely piloted vehicles,,$\sqrt{36}$ obstacle avoidance tasks,,$\frac{1}{1}$ and path following tasks $\frac{37}{37}$

In this experiment, haptic aids for pitch tracking task with compensatory display are investigated. The haptic cue was designed as a linear system that generates an additional force based on the visual error dynamics (HAPT block in figure 2). Both DHA and IHA approaches were analysed.

The aim of the design of the DHA was to develop a system that would try to mimic the pilot control strategy. In this way, the pilot should trust the haptic aid and be compliant with the haptic forces. If the visual error dynamics are such that the pilot should push the stick forward, DHA produces a force on the stick in the forward direction. Figure 10 shows an example of the haptic force given by a typical DHA.

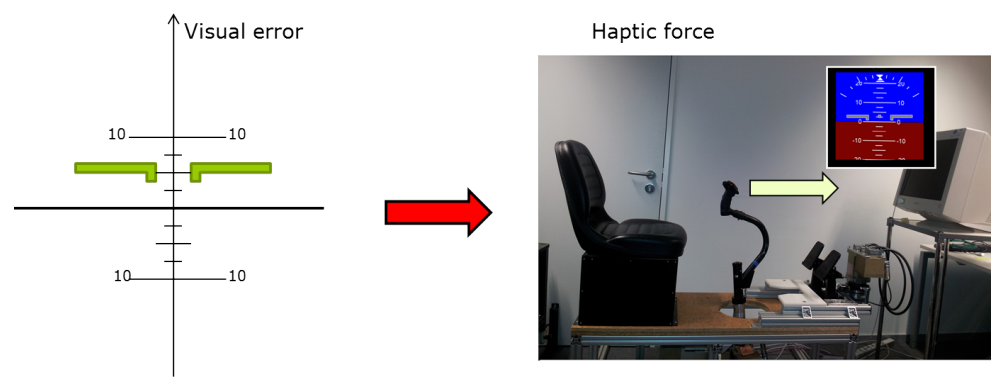

Figure 10. DHA approach for pitch tracking task. With a positive pitch error, the pilot has to push the stick forward, so does the haptic aid by generating the haptic force (yellow arrow).

The IHA was designed with the same dynamics of DHA, but opposite in sign (figure 11). This type of aid would result in a tendency of the aircraft to fly away from the tracking path if the pilot is not on the loop. In order to obtain a straight trajectory in that case, the indirect force feedback was changed in a shift of the neutral point of the stick. This approach has been already adopted by Alaimo ${ }^{1}$ and by De Stigter ${ }^{37}$ If the visual error dynamics need a control action in the forward direction, IHA will shift the neutral point in the backward direction and the pilot will feel a force in the direction shown in figure 11. If the pilot tries to oppose to the haptic forces, he/she will give a forward control action and the CE will follow the tracking path. On the contrary, without a pilot control action, the stick will change its deflection but the CE will continue to flight straight. This approach was found to be easier to exploit by pilots: even if the pilot thinks only to stop the stick in the centre, the neutral point shift given by IHA will generate a control action for the $\mathrm{CE}$, reaching good performances. 


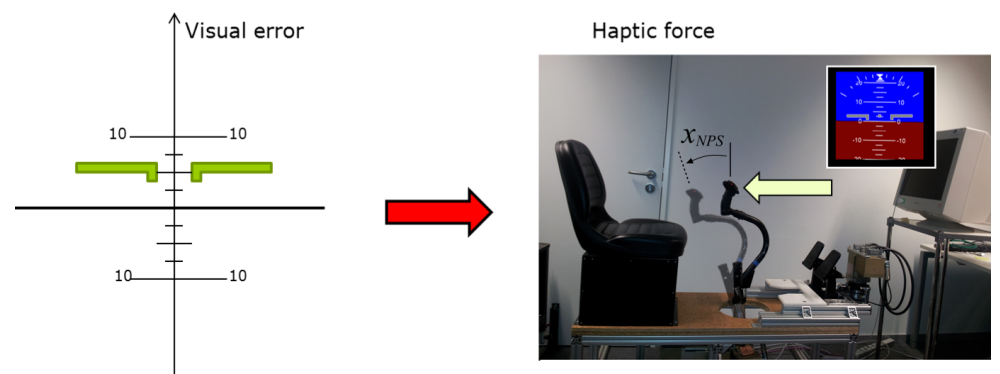

Figure 11. IHA approach for pitch tracking task. With a positive pitch error, the pilot has to push the stick forward, then the haptic aid generates a force that pulls the stick backward, and moves the neutral point backward as well. By this latter expedient no actual command is given by the aid to the aircraft without pilot intervention.

\section{Experiment}

\section{VI.A. Hypotheses}

The experiment aims at investigating the behaviour of the human pilot in relation with different haptic aids. The hypotheses were that:

1. the variation of the haptic aid has a significant effect on the admittance and the visual response of the pilot.

2. the performance and the effort of the pilots vary depending of the haptic aid.

3. ARX and CSD-ML methods give consistent admittance estimates. The admittance estimates given by CSD-based method is different if the assumption about the visual error $e$ is not fulfilled

\section{VI.B. Apparatus}

The experiment was performed using a control-loading system (WAS) from Wittenstein Aerospace \& Simulation GmbH, Germany. WAS includes a cyclic stick, a collective stick and directional pedals in order to replicate helicopter control (the WAS can be seen in figures 10 and 11). The devices can be used to simulate a wide range of control dynamics, and can provide force-feedback to the pilot, i.e. he/she can sense a force feedback in the end-effector. During the experiment, only the cyclic stick was used. The roll axis of the stick was fixed. No armrest was present for the arm that controlled the side stick. Subjects were asked not to use their legs as support.

The WAS was programmed to implement a mass spring damper system, and its transfer function $H_{C D}$ was obtained by frequency domain identification:

$$
H_{C D}(s)=\frac{1}{1.522 s^{2}+8.832 s+86.469} \quad[\mathrm{rad} / \mathrm{N}]
$$

Participants watched the primary flight display (PFD) shown in figure 12 For all the conditions, only the pitch angle varied. The display was refreshed with a rate of $100 \mathrm{~Hz}$.

All the components were driven by a real-time computer running xPC Target, which is a real time environment produced by MathWorks. The simulation ran at $100 \mathrm{~Hz}$, including the generation of the forcing functions and the data measurements.

\section{VI.C. Subjects}

Eight subjects participated in the experiment, seven males and one female. All participants were Ph.D. students at the Max Planck Institute, some had general experience with flight simulators and closed-loop control tasks. A financial compensation was offered for their participation.

\section{VI.D. Experiment protocol}

Before starting the experiment, the subjects received instructions about the objective of the experiment. The experiment was split in two different parts, the classical tasks and the tracking tasks. 


\section{Classical tasks}

The first part of the experiment allowed to quantify the range over which subjects could adapt their admittance. It consisted of admittance measurement while performing three different tasks in face of continuous disturbance force they could sense on the stick (so-called "classical tasks"):

1. relax task $(R T)$, in which participants were asked to relax their arm and adopt a passive behaviour. The display was turned off to prevent any distraction.

2. position task $(R T)$, in which participants were asked to minimize the stick deviation by resisting the disturbance force. The display showed the stick deviation.

3. force task $(F T)$, in which the task was to be compliant with the disturbance force and to minimize the force applied to the stick. During the first trials, the display showed the force applied to the stick. Only after some training, the display was switched off.

To avoid learning effects, the order of the tasks was randomized between subjects using a Latin square matrix ${ }^{38}$ Each condition was recorded at $100 \mathrm{~Hz}$, lasted $90 \mathrm{~s}$, and was repeated three times for averaging purposes. This study investigated stationary behaviour, and, in order to remove any transient effect and for the FFT analysis, some samples at the beginning and at the end were eliminated, resulting in 8192 samples for data analysis.

The disturbance forces were designed as explained in section III.A. The power of the disturbance forces was scaled in an attempt to obtain the same standard deviation of the stick position for all the classical tasks. This is considered important because it should let the NMS work approximately at the same operating points, thus avoiding non linearities. For all the tasks, the same RP gain (high frequency components) was used, whereas the FP gains (low frequency components) were chosen as 1.5, 2, and 10 times larger the RP gain for FT, RT, and PT respectively.

During the experiment, the force $F_{\text {hum }}$ applied by participants to the stick and the stick deflection $x_{C D}$ were measured. The measurements were averaged in the time domain over all the repetitions in order to reduce the noise. The human admittance was then estimated according to the method explained in section III.A.

\section{Tracking Task}

In the second part of the experiment, participants were asked to track the reference pitch signal as accurately as possible. The PFD was used as a compensatory display, showing the error $e$ between the reference pitch angle $x_{t a r}$ and the actual pitch angle of the aircraft. The task was to keep the error as close to zero as possible (figure 12 .

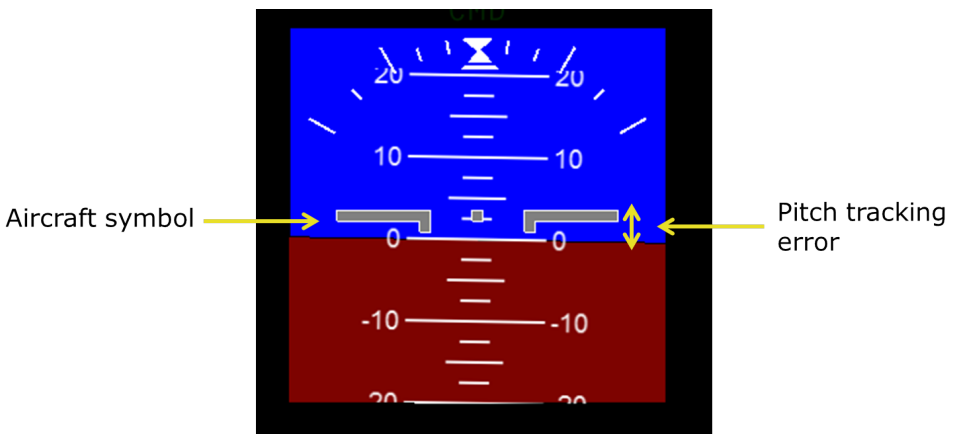

Figure 12. Primary flight director (PFD) during the tracking task. The error $e$ is showed in the pitch axis.

The system dynamics were chosen as:

$$
H_{C E}=6 \frac{3}{s(s+3)} \quad[\mathrm{rad} / \mathrm{rad}]
$$

Similar dynamics were used in previous studies $\frac{\sqrt[30]{30}}{2 t}$ higher frequencies, the selected dynamics is similar to those of a double integrator, that is not very easy to control for the pilot. This dynamics should accentuate the differences in pilot performance between different haptic aid conditions. 
While performing the task, a continuous force disturbance signal $F_{d i s t}$ was applied to the stick in order to estimate the admittance of the arm. The two forcing signals $F_{d i s t}$ and $x_{t a r}$ were designed as explained in section III.B. During the tracking task, the force disturbance signal $F_{\text {dist }}$ was tuned in order to reduce unwanted effects on the neuromuscular system while still allowing good signal-to-noise ratio (for the estimators); the resulting RP gain was 1.3 times larger the RP value used in the classical tasks, while the resulting normalized FP gain was 1.5.

Haptic aids were used to help the participants during the tracking task. Three different cases were investigated:

1. no haptic aid (NoHA): no haptic aid was used during tracking task. Together with force disturbance, the pilot felt the WAS stiffness and damping only.

2. direct haptic aid (DHA): the haptic aid suggested the right control action. Participants should be compliant in order to benefit from the haptic aid.

3. indirect haptic aid (IHA): the haptic aid consisted in a force in the opposite direction. Participants should oppose to the force in order to achieve the task.

The two haptic aids were designed with the same dynamics but opposite in sign:

$$
H_{\text {hapt }}^{D H A}=-H_{\text {hapt }}^{\text {IHA }}=-13 \frac{s+3}{0.05 s+1} \quad[N / \mathrm{rad}]
$$

In the case of IHA only, $x_{N P S}$ was set to:

$$
x_{N P S}^{I H A}=H_{\text {hapt }}^{\text {IHA }}(s) H_{C D}(s) e
$$

while $x_{N P S}=0$ in the other cases. This dynamics was chosen in order to mimic the well documented 17 pilot control behaviour during a pitch tracking task without haptic aid. The aim was to replicate the human control strategy, such that participants could trust the haptic aid and be compliant with it during the DHA approach. Subjects did not know the type of haptic aid they were going to use. Each condition was repeated 8 times for averaging purposes. The order of the tasks and the time duration of the repetitions were designed in the same way as for the classical tasks.

During the experiment, the force $F_{h u m}$ applied by participants to the stick, the stick deflection $x_{C D}$, and the visual error $e$ were measured. The measured signals were averaged over all the repetitions, in order to reduce the noise. The arm admittance, the visual response, and the open-loop transfer function were then estimated using all the three identification methods presented in section III.B.

\section{Results and discussion}

\section{VII.A. Performance Parameters}

Figure 13 shows the target pitch angle $x_{t a r}$ and pitch angle of the controlled element $x_{C E}$ measured during NoHA, DHA and IHA cases (figure shows results for a sample participant).

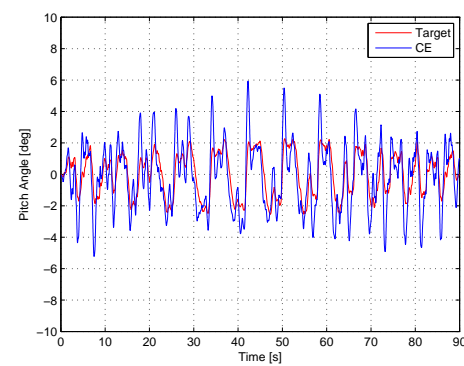

(a) NoHA.

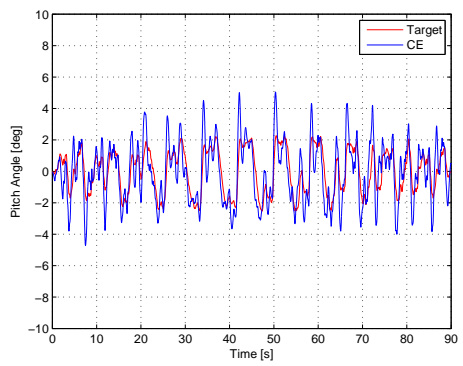

(b) DHA

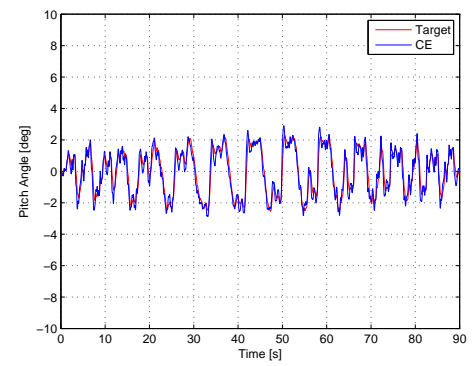

(c) IHA.

Figure 13. Time realisations of target and CE pitch angles relating to the subject 4 . 
To quantify changes in the pilot control behaviour in the various experimental conditions, the root means square error (RMS) of time signal realisations was calculated. The RMS of a generic time signal $r$ is defined as:

$$
R M S=\sqrt{\frac{\sum_{k=1}^{N} r(k)^{2}}{N}}
$$

where $\mathrm{N}$ represents the number of time samples.

Figure 14 presents the RMS values and the $95 \%$ confidence intervals of the visual error $e=x_{t a r}-x_{C E}$, the human force $F_{\text {hum }}$, and the stick deflection $x_{C D}$ averaged over all the participants, that can be considered as a measure of performance.

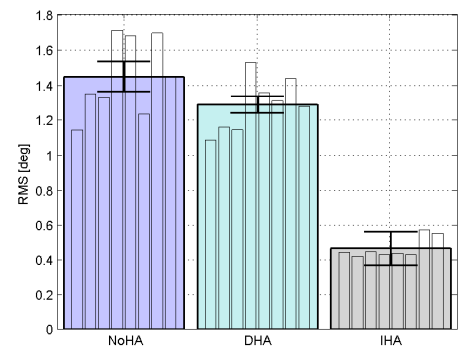

(a) Visual error $e$.

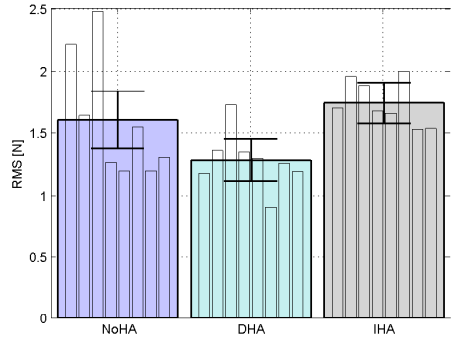

(b) Human force $F_{\text {hum }}$.

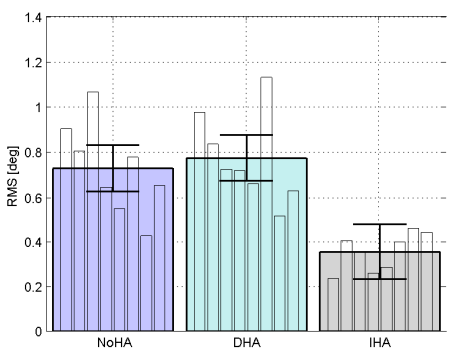

(c) Control device deflection $x_{C D}$.

Figure 14. Means and $95 \%$ confidence intervals of $R M S s$ over all the participants.

Using an ANOVA with repeated measures, the mean scores for the RMS of the visual error were statistically significantly different $(F(2,14)=162.950, p<0.05)$. Post hoc tests using the Bonferroni correction revealed that there was a significant difference between DHA and IHA and between NoHA and IHA $(p<0.05)$. The tests also indicated a significant difference between NoHA and DHA $(p=0.021)$. The results showed a large increase in the performances with the IHA. As already discussed, the IHA system provides most of the control action. Even if the pilot does not make an active control action and tries only to hold the stick in the centre, he can reach good performance. With DHA, the RMS of the visual error significantly decreased with respect to NoHA as well. This indicates that both IHA and DHA help the pilot to perform better during the tracking tasks.

Considering the RMS of the human force, the ANOVA test indicated that there was a statistically significantly difference between different haptic aids $(F(2,14)=5,742, p=0.015)$. A post-hoc test with Bonferroni correction revealed that the reduction in the human force from NoHa to DHA and the increase from NoHA to IHA were not statistically significant (respectively, $p=0.226$ and $p=1$ ). A statistically significant effect could be found instead between two groups, the IHA and the DHA $(p=0.009)$. During DHA the operators were more compliant and applied less force to the stick respect to IHA case. This indicates that less control effort is needed to achieve the task.

For the RMS of the stick deflection, the test showed a main effect of the type of haptic aid $(F(2,14)=$ $16.140, p<0.05)$. Post-hoc tests with Bonferroni correction indicated a statistically significant difference between the IHA and the other two cases ( $p=0.010$ and $p=0.005$ in the comparison with NoHA and DHA respectively). This means that, even though participants apply larger forces during IHA, the stick motion is smaller since the participants tend not to move the stick in a large range, but only to oppose to the haptic force; the result is less Work of the pilot in terms of control activity. Despite that, the IHA condition is rather tiring for the pilots, because they constantly have to co-contract their muscles.

\section{VII.B. Admittance}

\section{Classical Tasks}

Figure 15 shows the admittance measured during classical tasks. The admittances was averaged over all the participants, and the shaded areas show the standard deviation. As expected, the admittance was higher in the force task, because the subject was asked to be compliant with the disturbance function. Again as expected, the subjects were stiffer in the position task, because they tried to oppose to the forces they felt in 
the stick. During the relax task, as known from literature, the humans adopted a passive behaviour and the admittance lay between the other two tasks. At higher frequencies, the differences became smaller, because the arm dynamics are more and more governed by the inertia. High squared coherences were found at all frequencies for all tasks. The results were found to be comparable with the results of previous studies $11 \mid 27$

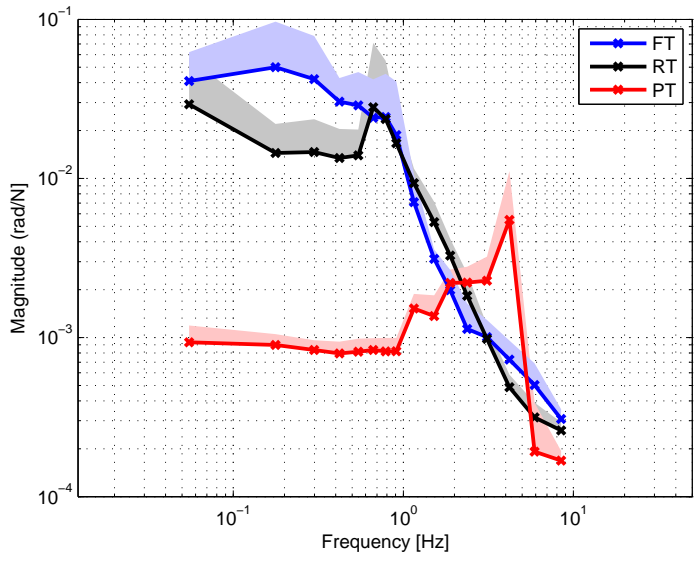

(a) Admittance.

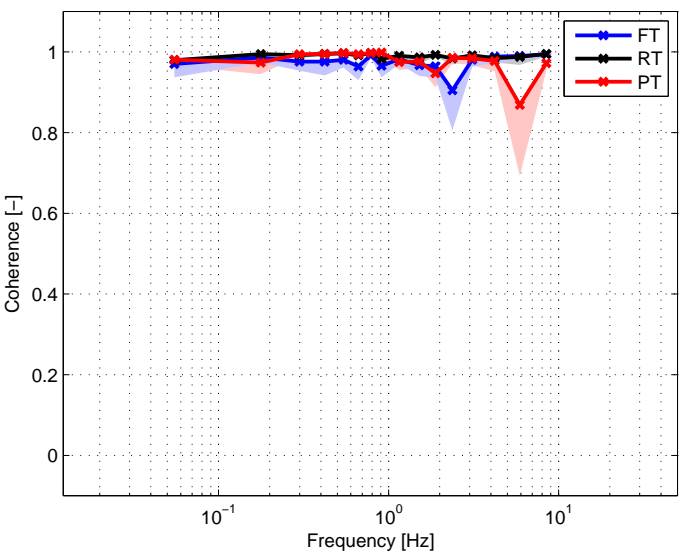

(b) Coherence function.

Figure 15. Admittance frequency response functions and coherence functions measured during force $(F T)$, position $(P T)$ and relax tasks $(R T)$. The lines shows the magnitude of admittance averaged over all subjects, the shadows show the standard deviation.

\section{Tracking Task}

The human admittance during tracking tasks with different haptic aids was estimated using the three different methods explained in section III.B. We expected the participants to be able to adapt their neuromuscular settings depending on the provided haptic aid.

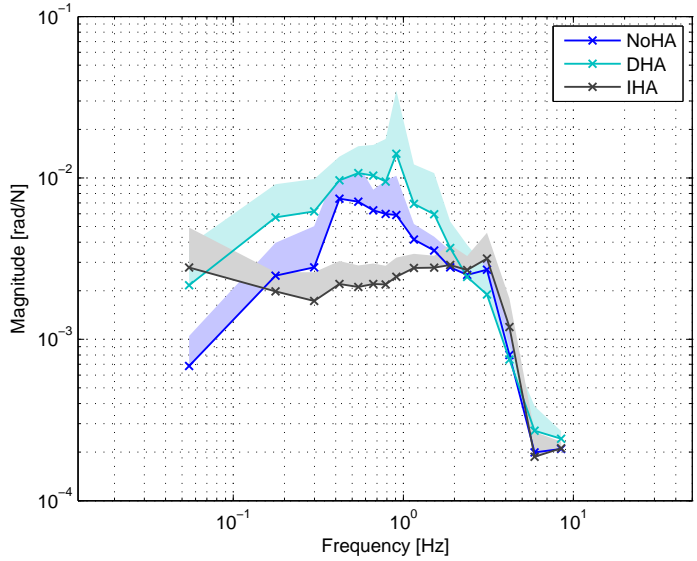

(a) Admittance.

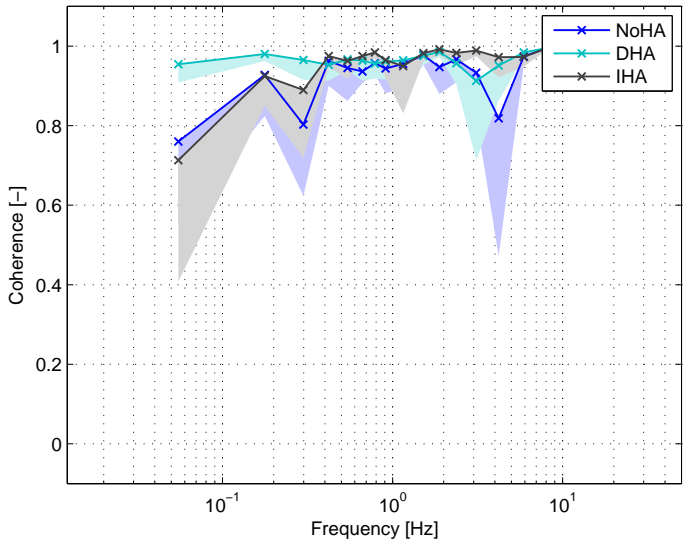

(b) Coherence function.

Figure 16. Admittance frequency response functions and coherence functions measured during pitch tracking task without haptic aid $(N o H A)$, with direct haptic aid (DHA) and indirect haptic aid (IHA). The estimates are averaged over all participants, the shadows show the standard deviation.

Figure 16 shows the admittance estimations given by the CSD method. The admittances were averaged over all eight subjects. High squared coherence values were found at all frequencies for all haptic aids, justifying the linearity assumption of the model. The figure shows a clear dependence of the subject admittance on the type of haptic aid. With DHA the humans were compliant with haptic forces, designed in order to suggest the right control action (higher levels of admittance as in the force task). With IHA the subjects tried to oppose to the haptic forces and became stiffer. The admittance during the NoHA case attained values between the two previous cases; the neuromuscular system was more stiff than with DHA and less stiff 
than with IHA. These results support the hypothesis that the humans are able to adapt their neuromuscular system in response to the type of haptic aid.

As seen in section III.B the CSD identification method assumes that the visual error $e$ does not contain power at frequencies $f_{d}$ where the disturbance force contains power. Figure 17 shows the PSD of the visual error $e$ during NoHA tracking taks. Similar PSDs were obtained for DHA and IHA cases.

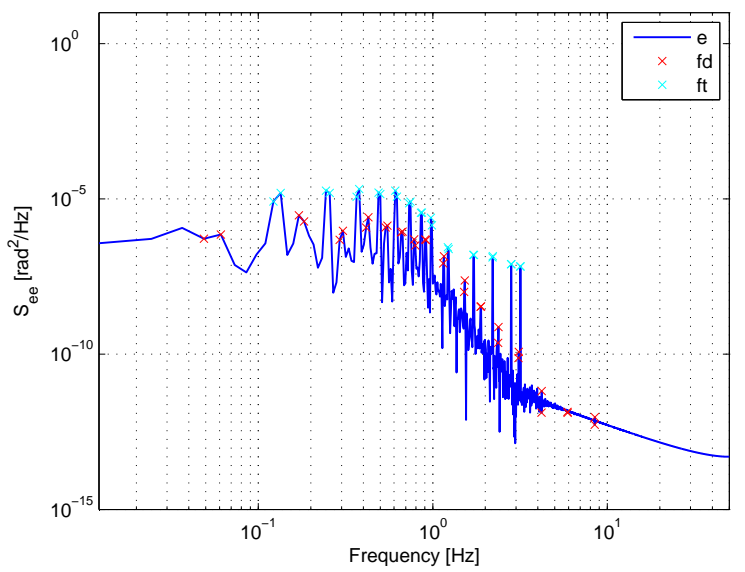

Figure 17. PSD of the visual error of subject 3 during NoHA task. The crosses represent the disturbance force and target position frequencies.

The necessary assumption, for the CSD-based method, is clearly not satisfied at low frequencies, where the power of $e$ presents high peaks at the $f_{d}$ frequencies (while it should have peaks at $f_{t}$ frequencies only). Thus, we can assume that the participants saw these frequencies on the visual display and tried cognitively to respond to them. Therefore, the human force at $f_{d}$ frequencies is not given only by the arm response $F_{a d m}$ to the disturbance forces, but contains also components from the visual system $F_{v i s}$. Thus, the admittance estimates are probably corrupted by this wrong assumption; since the PM and CSD-ML methods do not make assumptions about the power content of the visual error and consider the pilot as a multi-loop system, we expect that ARX and CSD-ML methods should give low-frequencies admittance estimates different from those obtained by the CSD-based method, and consistent between them.

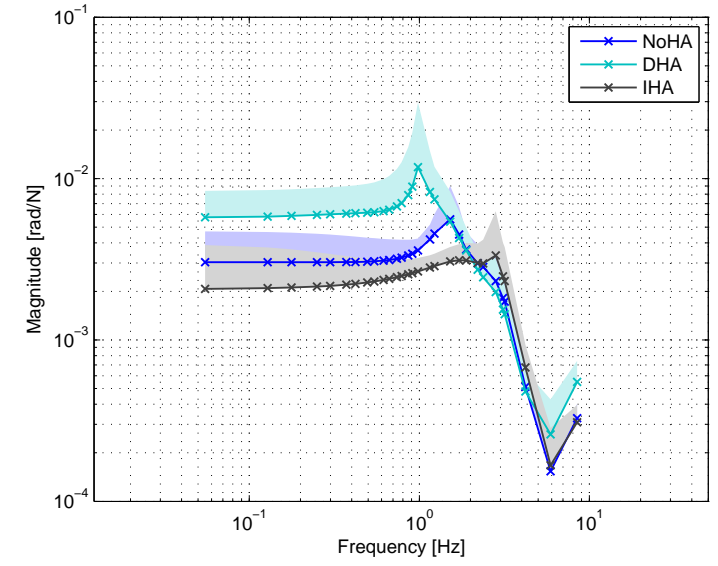

(a) ARX models.

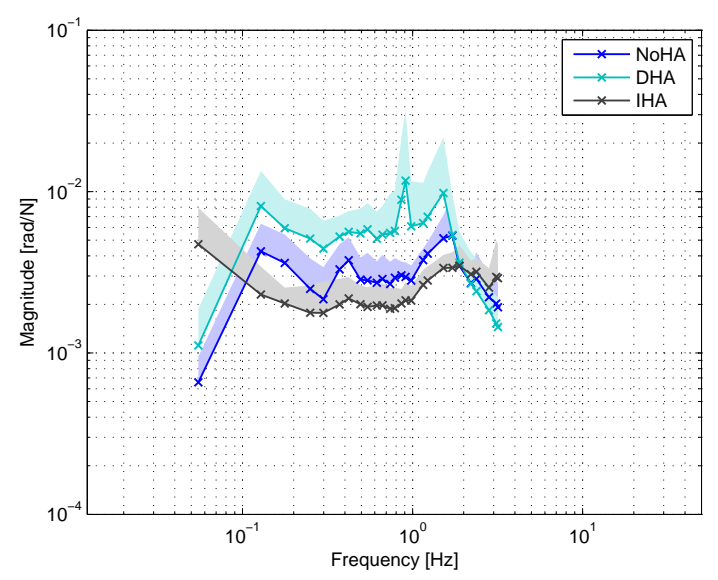

(b) Multi-loop cross-spectral density estimates.

Figure 18. Admittance frequency response functions from ARX and CSD-ML methods.

Figure 18 shows the admittance estimates given by the two methods. It should be noted that CSD-ML method gives admittance FRFs only below $3 \mathrm{~Hz}$, that is the highest value in the $f_{t}$ frequencies. For ARX models, the FRFs can be calculated at all the desired frequencies $\left(f_{d}, f_{t}\right)$. The VAF can be calculated as an indicator of reliability of ARX estimates. The VAF values averaged over all the participants are high for all the tracking tasks $\left(V A F_{D H A}=90.5 \%, V A F_{I H A}=72 \%\right.$, and $\left.V A F_{N o H A}=87 \%\right)$. This means that ARX 
models can predict the measured output signal well. For CSD-ML there is no indicator for the goodness of the estimates. Both identification methods pointed out the same admittance behaviour found with CSDbased method: the pilot becomes stiffer with IHA, and more compliant with DHA with respect to the NoHA case.

Figure 19 shows a comparison between admittance from the three identification methods. As expected, the admittance estimates given by ARX and CSD-ML are different from those given by CSD technique especially at lower frequencies. Only for IHA case the three methods give almost identical results. On the other hand, ARX and CSD-ML estimates appear quite consistent by inspection of figure 19. CSD-ML estimate differs significantly from that of ARX for the first frequency point only. According to section III.B. this is due to the extrapolation procedure applied at this frequency point. The differences between the admittance estimates given by the three methods are comparable with those found in simulations (see section IV. At low frequencies $(f<0.2 \mathrm{~Hz})$, the magnitude of the admittance estimated by CSD technique is lower than the magnitude given by the other two methods. The opposite occurs at medium frequencies $(0.2 \mathrm{~Hz}<f<2 \mathrm{~Hz})$, whereas CSD and ARX methods give consistent estimates at higher frequencies.

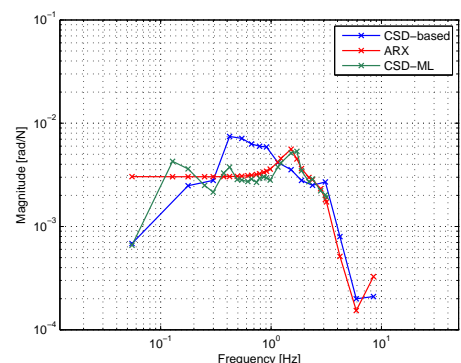

(a) NoHA.

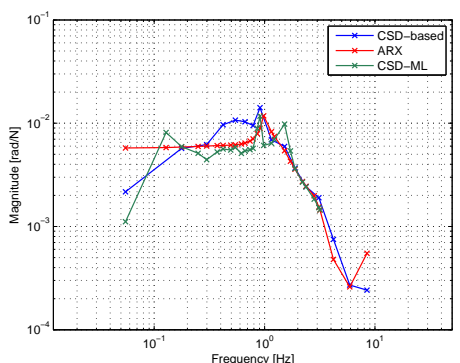

(b) DHA.

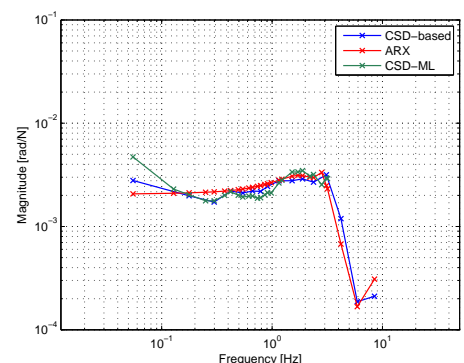

(c) IHA.

Figure 19. Comparison between admittance estimates given by the three different identification methods.

These observations suggest that during a tracking task the admittance estimate given by CSD method could be corrupted if the contribution of the visual response at frequencies $f_{d}$ on the human force cannot be neglected. The pilot model is a closed-loop system and the influence of the visual system must be taken in account during the admittance estimate.

Figure 20 shows the admittances as measured during the three pitch tracking tasks, together with those measured during classical tasks. The admittances of all conditions of pitch tracking were between the position and relax task. A similar result, for a tracking task but without haptic aids, was documented in a previous study 11 The admittances of all tasks converged at frequencies above $10 \mathrm{~Hz}$, where the inertial properties dominate the arm dynamics. At lower frequencies, subjects showed a substantial adaptation range of their admittances.

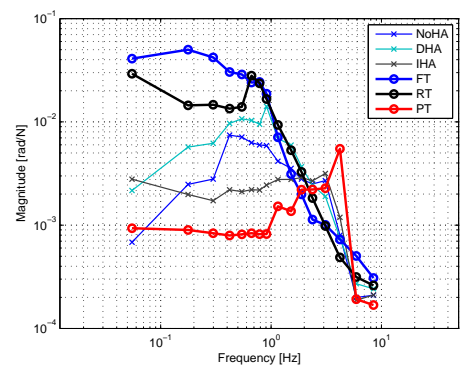

(a) CSD-based.

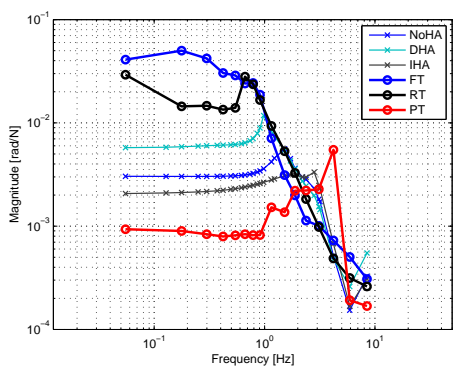

(b) ARX.

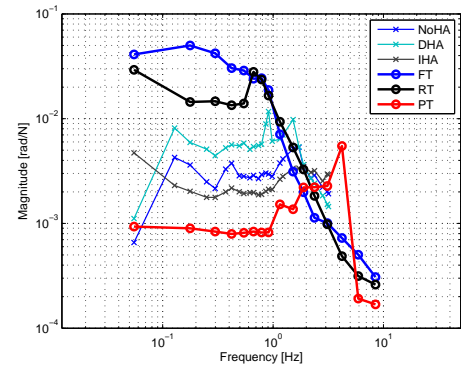

(c) CSD-ML.

Figure 20. Admittance frequency response functions for classical and tracking tasks.

\section{VII.C. Visual Response}

As explained in section III.B, only ARX and CSD-ML methods allow to compute directly an estimate for the pilot visual response. Figure 21 shows the visual response estimates obtained by ARX and CSD-ML 
methods. CSD-ML allows estimation only below $3 \mathrm{~Hz}$. Also the FRFs are by ARX is shown below $3 \mathrm{~Hz}$.

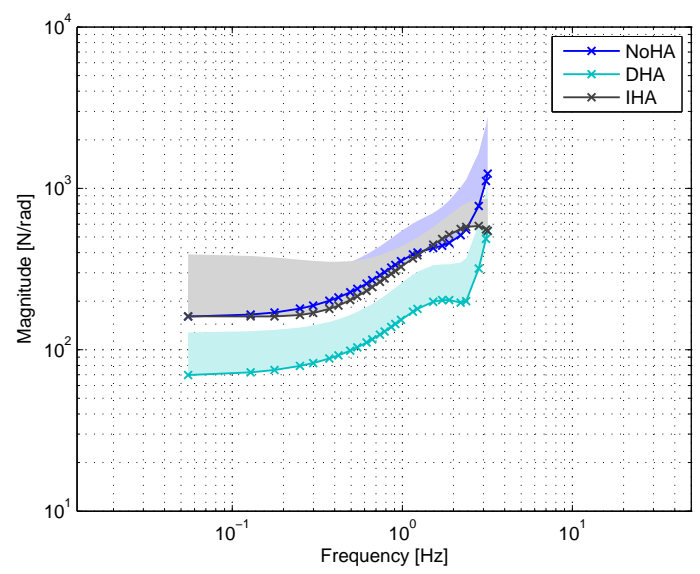

(a) ARX.

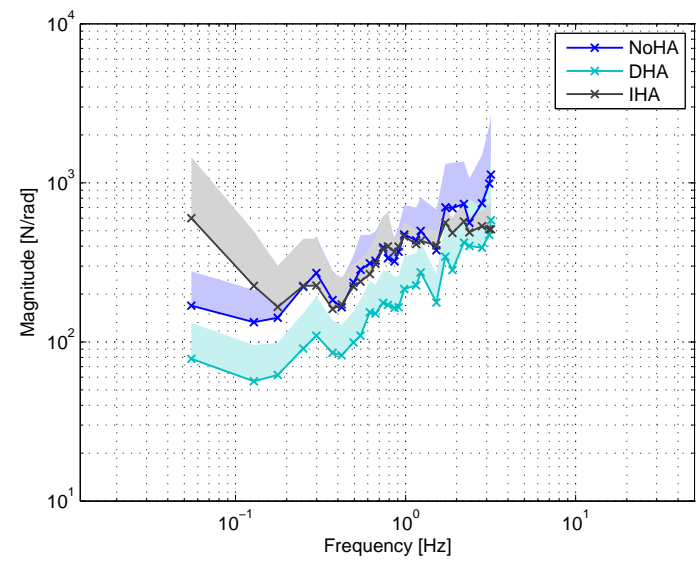

(b) CSD-ML.

Figure 21. Visual frequency response functions from ARX and CSD-ML methods.

The general shape of the visual responses is very similar for all the different conditions (NoHA, DHA, and IHA). Apparently participants did not change the dynamics of their response in relation with different haptic aids, but they vary only the gain.

The gain of the visual response is lower for DHA at all frequencies. This is probably due to the "direct" nature of the aid, that provides part of the action needed by the pilot. The pilot is compliant and applies less force with respect to the NoHA case.

It's interesting to note that the gain for IHA is comparable with the gain for NoHA. The haptic aid supports the pilots with forces opposite in sign respect to the DHA and complemented by the neutral point shift; thus the pilot can oppose to the haptic forces and apply a control action to the CD only adopting stiff settings for the arm neuromuscular system. This implies that most of the control action can be generated indirectly by the combination of the IHA system and low values of arm admittance. This control action still generates a visual error; the pilot response to this visual error is similar to the NoHA case.

As shown in figure 22, the two different identification methods give consistent results.

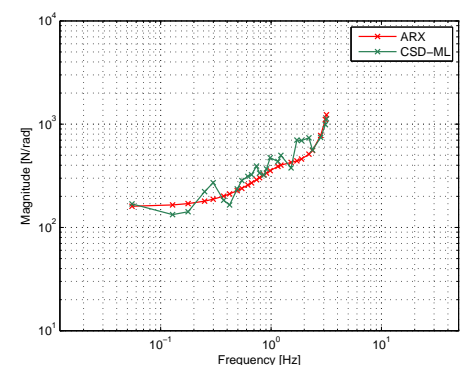

(a) NoHA.

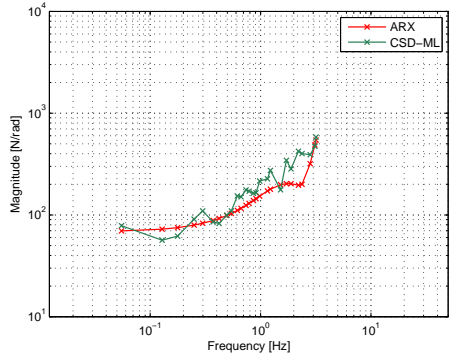

(b) DHA.

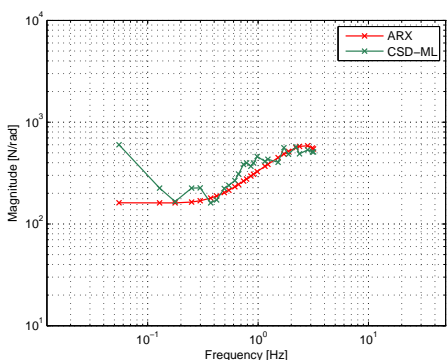

(c) IHA.

Figure 22. Comparison between visual system estimates given by ARX and CSD-ML identification methods.

\section{VII.D. Open-loop Transfer Function}

The open-loop transfer function $H_{O L}$ describes the transfer function from the visual error $e$ to the position of the controlled element $x_{C E}$ and includes the VIS, HAPT, ADM, CD and CE dynamics. Figure 23 shows the open-loop frequency response functions during the tracking tasks with different haptic aids. It should be noted that both CSD-based and CSD-ML methods estimate the open-loop FRF below $3 \mathrm{~Hz}$, that is the maximum frequency point in $f_{t}$, whereas the FRFs of ARX estimates can be calculated at any frequencies, therefore figure 23 shows only the FRF values below $3 \mathrm{~Hz}$ also for the ARX method. 


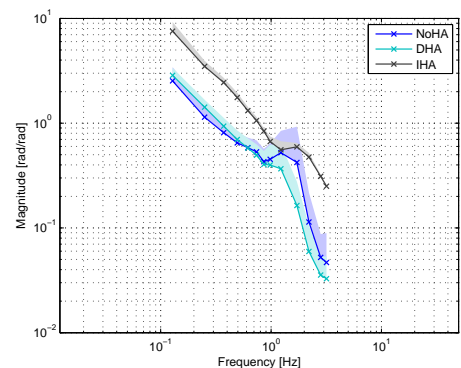

(a) CSD-based.

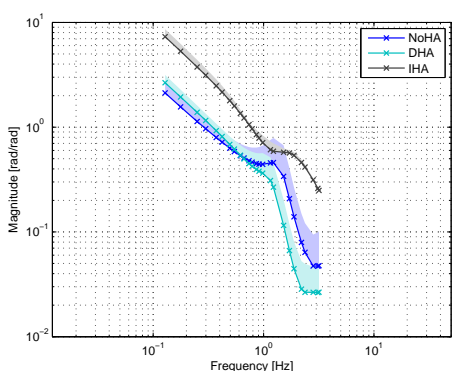

(b) ARX

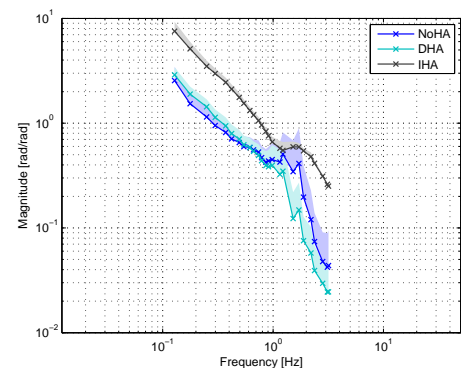

(c) CSD-ML.

Figure 23. Open-loop frequency response functions from the three identification methods.

According to McRuer theories, 17 the humans adapt their dynamics to those of the controlled element, in order to obtain combined pilot-vehicle dynamics that approximate those of a single integrator around the crossover frequency $\omega_{c}$. These theories were obtained from investigation into pilot control behaviour for single-loop tracking task without haptic aid. For all the tested haptic aids, the pilots adopted a single integrator-like behaviour around the crossover frequency, but we noticed a significant variation of the crossover frequency for IHA with respect to both DHA and NoHA.

Figure 24 shows the mean and the $95 \%$ confidence interval of the participant's crossover frequency values. The crossover frequency is statistically significantly different in the three different situations $(F(2,14)=$ 138.31, $p<0.05)$. A post-hoc test with Bonferroni correction showed that this significant effect can be found between the IHA case and the other haptic aids $(p<0.05)$. The apparent small increase of $\omega_{c}$ from NoHA to DHA is not statistically significant. This means that even though the pilot was more compliant with DHA (section VII.B), he/she adapted the visual system settings to obtain overall behaviour similar in both cases. The large difference between IHA and the other two cases can by explained, in our opinion, by the fact that IHA support system was found easier to be exploited by the test pilots: in most situations, if the human did not make any active control action and only held the stick in the centre, allowed the pilot to reach good performance. The IHA gives an important contribution on the open-loop transfer function. Figure 25 shows that the three identification methods gave consistent estimates of the open-loop transfer function.

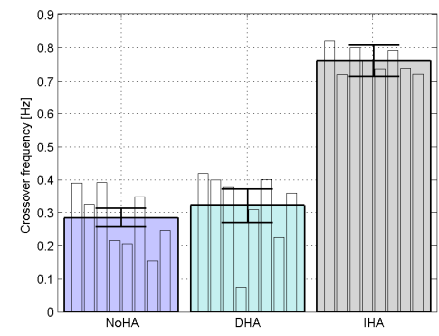

Figure 24. Means and $95 \%$ confidence intervals of the crossover frequency values.

\section{Conclusion}

The paper has presented a novel approach for multi-loop identification of pilot admittance and visual response while performing a tracking task with a compensatory display and haptic stick. The technique was first validated with simulations using pre-defined models, then experimental tests are presented with two different haptic aids based on the DHA and IHA approaches. The results show that the proposed identification techniques are a valid alternative to other techniques, as they do not require assumptions that limit their applicability or freedom of experiment design. Furthermore the paper shows that for aircraft pitch angle tracking the IHA-based controller outperforms the DHA in terms of reference tracking error. The open loop bandwidth of the whole system, including pilot, was found to be significantly larger than with DHA 


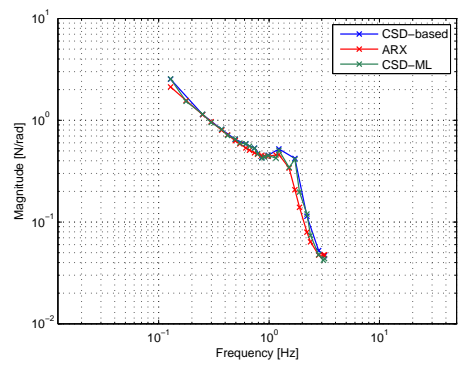

(a) NoHA.

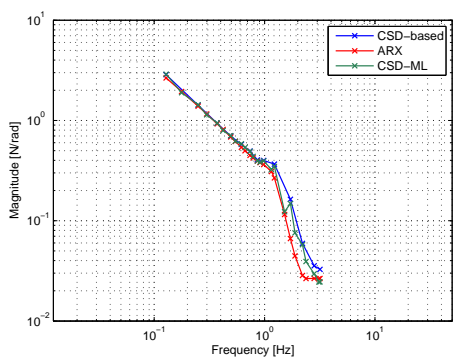

(b) DHA.

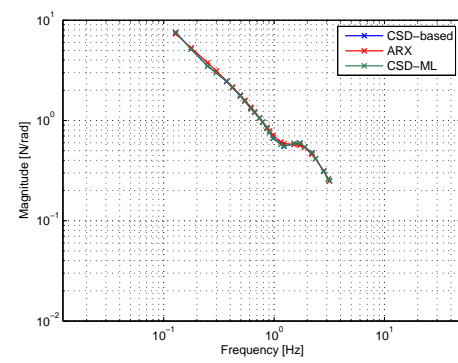

(c) IHA.

Figure 25. Comparison between pilot open-loop frequency response functions given by the three identification methods.

and NoHA motivating the significantly better tracking performance seen during the experiments. As shown in this paper, this change was the result of pilots changing their admittance from compliant to stiff when they were provided with a IHA as compared to an DHA. The increased performance with IHA came at the cost of slightly increased RMS of human force but complemented by a decreased RMS stick deflection.

\section{Acknowledgments}

The work in this paper was partially supported by the myCopter project, funded by the European Commission under the 7th Framework Program.

\section{References}

${ }^{1}$ Alaimo, S., Pollini, L., Bresciani, J.-P., and Bülthoff, H., "Evaluation of Direct and Indirect Haptic Aiding in an Obstacle Avoidance Task for Tele-Operated Systems," Proceedings of the 18th World Congress of the International Federation of Automatic Control (IFAC 2011), International Federation of Automatic Control, 9 2011, pp. 1-6.

${ }^{2}$ Alaimo, S., Pollini, L., Magazzù, A., Bresciani, J.-P., Robuffo Giordano, P., Innocenti, M., and Bülthoff, H., "Preliminary Evaluation of a Haptic Aiding Concept for Remotely Piloted Vehicles," Haptics: Generating and Perceiving Tangible Sensations (EuroHaptics 2010), Springer, Berlin, Germany, 7 2010, pp. 418-425.

${ }^{3}$ Lam, T., Boschloo, H., Mulder, M., and van Paassen, M., "Artificial Force Field for Haptic Feedback in UAV Teleoperation," Systems, Man and Cybernetics, Part A: Systems and Humans, IEEE Transactions on, Vol. 39, No. 6, nov. 2009, pp. $1316-1330$.

${ }^{4}$ de Vlugt, E., Identification of Spinal Reflexes, Ph.D. thesis, Delft University of Technology, Faculty of Mechanical Engineering, 2004.

${ }^{5}$ De Vlugt, E., Schouten, A. C., and Van Der Helm, F. C. T., "Quantification of intrinsic and reflexive properties during multijoint arm posture." Journal of Neuroscience Methods, Vol. 155, No. 2, 2006, pp. 328-349.

${ }^{6}$ Mugge, W., Abbink, D. A., Schouten, A. C., DeWald, J. P. A., and van der Helm, F. C. T., "A rigorous model of reflex function indicates that position and force feedback are flexibly tuned to position and force tasks," Experimental Brain Research, Vol. 200, 2010, pp. 325-340.

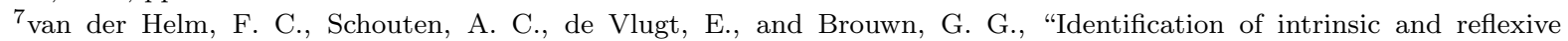
components of human arm dynamics during postural control," Journal of Neuroscience Methods, Vol. 119, No. 1, 2002, pp. 1 -14 .

${ }^{8}$ Schouten, A., de Vlugt, E., van Hilten, J., and van der Helm, F., "Quantifying Proprioceptive Reflexes During Position Control of the Human Arm," Biomedical Engineering, IEEE Transactions on, Vol. 55, No. 1, jan. 2008, pp. 311 -321.

${ }^{9}$ Schouten, A., Vlugt, E., and van der Helm, F., "Design of perturbation signals for the estimation of proprioceptive reflexes." IEEE Trans Biomed Eng, Vol. 55, No. 5, 2008, pp. 1612-9.

${ }^{10}$ Damveld, H. J., Abbink, D. A., Mulder, M., Mulder, M., van Paassen, M. M., van der Helm, F. C. T., and Hosman, R. J. A. W., "Measuring the Contribution of the Neuromuscular System During a Pitch Control Task," Proceedings of the AIA A Modeling and Simulation Technologies Conference, Chicago, Illinois, Aug. 10-13, 2009, No. AIAA-2009-5824, American Institute of Aeronautics and Astronautics, Aug. 2009.

${ }^{11}$ Damveld, H. J., Abbink, D. A., Mulder, M., Mulder, M., van Paassen, M. M., van der Helm, F. C. T., and Hosman, R. J. A. W., "Identification of the Feedback Component of the Neuromuscular System in a Pitch Control Task," Proceedings of the AIAA Guidance, Navigation, and Control Conference 2 - 5 August 2010, Toronto, Ontario Canada, edited by M. Silvestro, No. AIAA 2010-7915, American Institute of Aeronautics and Astronautics, American Institute of Aeronautics and Astronautics, Aug. 2010, pp. 1-22.

${ }^{12}$ Abbink, D. A., Neuromuscular Analysis of Haptic Gas Pedal Feedback during Car Following, Ph.D. thesis, Delft University of Technology, Faculty of Mechanical Engineering, Dec. 2006.

${ }^{13}$ Alaimo, S., Pollini, L., Bresciani, J.-P., and Bülthoff, H., "A Comparison of Direct and Indirect Haptic Aiding for Remotely 
Piloted Vehicles," Proceedings of the 19th IEEE International Symposium in Robot and Human Interactive Communication (IEEE Ro-Man 2010), IEEE, Piscataway, NJ, USA, 9 2010, pp. 506-512.

${ }^{14}$ McRuer, D. and Weir, D., "Theory of Manual Vehicular Control," Ergonomics, Vol. 12, No. 4, 1969, pp. 599-633.

${ }^{15}$ Hess, R. A., "Pursuit Tracking and Higher Levels of Skill Development in the Human Pilot," IEEE Transactions on Systems, Man, and Cybernetics, Vol. SMC-11, No. 4, 1981, pp. 262-273.

${ }^{16}$ McRuer, D., Human Pilot Dynamics in Compensatory Systems: Theory, Models and Experiments with Controlled Element and Forcing Function Variations, Technical report (Air Force Flight Dynamics Laboratory (U.S.))), Air Force Flight Dynamics Laboratory, Research and Technology Division, Air Frorce Systems Command, 1965.

${ }^{17}$ McRuer, D. and Jex, H., "A Review of Quasi-Linear Pilot Models," Human Factors in Electronics, IEEE Transactions on, 1967 .

${ }^{18}$ Nieuwenhuizen, F. M., Zaal, P. M. T., Mulder, M., van Paassen, M. M., and Mulder, J. A., "Modeling Human Multichannel Perception and Control Using Linear Time-Invariant Models," Journal of Guidance, Control, and Dynamics, Vol. 31, No. 4, July-August 2008, pp. 999-1013.

${ }^{19}$ Pool, D. M., Zaal, P. M. T., Damveld, H. J., van Paassen, M. M., van der Vaart, J. C., and Mulder, M., "Modeling WideFrequency-Range Pilot Equalization for Control of Aircraft Pitch Dynamics," Journal of Guidance, Control, and Dynamics, Vol. 34, No. 5, September-October 2011, pp. 1529-1542.

${ }^{20}$ van Paassen, M. M., Biophysics in Aircraft Control, A Model of the Neuromuscular System of the Pilot's Arm, Ph.D. thesis, Delft University of Technology, Faculty of Aerospace Engineering, 1994.

${ }^{21}$ Abbink, D. A., Mulder, M., van der Helm, F. C. T., Mulder, M., and Boer, E. R., "Measuring neuromuscular control dynamics during car following with continuous haptic feedback." IEEE transactions on systems, man, and cybernetics. Part B, Cybernetics, Vol. 41, No. 5, 2011, pp. 1239-1249.

${ }^{22}$ Abbink, D. A. and Mulder, M., Neuromuscular Analysis as a Guideline in designing Shared Control, InTech, April 2010, pp. 499-416.

${ }^{23}$ Pintelon, R. and Schoukens, J., System identification: a frequency domain approach, IEEE Press, 2001.

${ }^{24}$ Mugge, W., Abbink, D. A., and Van Der Helm, F. C. T., "Reduced Power Method: how to evoke low-bandwidth behaviour while estimating full-bandwidth dynamics," 2007 IEEE 10th International Conference on Rehabilitation Robotics, Vol. 00, No. c, 2007, pp. 575-581.

${ }^{25}$ van Lunteren, A., Identification of Human Operator Describing Function Models with One or Two Inputs in Closed Loop Systems, Ph.D. thesis, Delft University of Technology, Faculty of Mechanical Engineering, Jan. 1979.

${ }^{26}$ Zaal, P. M. T., Pool, D. M., Chu, Q. P., van Paassen, M. M., Mulder, M., and Mulder, J. A., "Modeling Human Multimodal Perception and Control Using Genetic Maximum Likelihood Estimation," Journal of Guidance, Control, and Dynamics, Vol. 32, No. 4, 2009, pp. 1089-1099.

${ }^{27}$ Venrooij, J., Abbink, D., Mulder, M., and van Paassen, M., "A Method to Measure the Relationship Between Biodynamic Feedthrough and Neuromuscular Admittance," Systems, Man, and Cybernetics, Part B: Cybernetics, IEEE Transactions on, Vol. 41, No. 4, aug. 2011, pp. $1158-1169$.

${ }^{28}$ Ljung, L., System Identification: Theory for the User, Pearson Education, 1998.

${ }^{29}$ Pintelon, R., Guillaume, P., Rolain, Y., Schoukens, J., and Van Hamme, H., "Parametric identification of transfer functions in the frequency domain-a survey," Automatic Control, IEEE Transactions on, Vol. 39, No. 11, nov 1994, pp. 2245 -2260 .

${ }^{30}$ Hess, R., "Modeling the effects of display quality upon human pilot dynamics and perceived vehicle handling qualities," Systems, Man and Cybernetics, IEEE Transactions on, Vol. 25, No. 2, feb 1995, pp. 338 -344.

${ }^{31}$ Diolaiti, N. and Melchiorri, C., "Teleoperation of a mobile robot through haptic feedback," Haptic Virtual Environments and Their Applications, IEEE International Workshop 2002 HAVE, 2002, pp. 67 - 72.

${ }^{32}$ Burdea, G. C., Force and touch feedback for virtual reality, John Wiley \& Sons, Inc., New York, NY, USA, 1996.

${ }^{33}$ Cummings, M., Donmez, B., and Graham, H., "Assessing the Imact of Haptic Peripheral Displays for UAV Operators," Technical report, MIT Humans and Automation Laboratory, 2008.

${ }^{34}$ Lam, T., Mulder, M., van Paassen, M., and van der Helm, F., "Tele-operating a UAV using haptics - modeling the neuromuscular system," Systems, Man and Cybernetics, 2005 IEEE International Conference on, Vol. 3, oct. 2005, pp. 2695 -2700 Vol. 3.

${ }^{35}$ Lam, T., Mulder, M., and van Paassen, M., "Haptic interface in UAV tele-operation using force-stiffness feedback," Systems, Man and Cybernetics, 2009. SMC 2009. IEEE International Conference on, oct. 2009, pp. 835 -840.

${ }^{36}$ Alaimo, S., Pollini, L., Bresciani, J.-P., and Bülthoff, H., "Augmented Human-Machine Interface: Providing a Novel Haptic Cueing to the Tele-Operator," Proceedings of the 3rd Workshop for Young Researchers on Human-Friendly Robotics (HFR 2010), 10 2010, pp. 1-2.

${ }^{37}$ de Stigter, S., Mulder, M., and Van Paassen, M., "Design and evaluation of haptic flight director." Journal of guidance, control and dynamics, Vol. 30, No. 1, 2007, pp. 35-46.

${ }^{38}$ Dénes, J. and Keedwell, A. D., Latin squares, Vol. 46 of Annals of Discrete Mathematics, North-Holland Publishing Co., Amsterdam, 1991. 